\title{
Nonsense-mediated mRNA decay: An intricate machinery that shapes \\ transcriptomes
}

\author{
Søren Lykke-Andersen and Torben Heick Jensen \\ Centre for mRNP Biogenesis and Metabolism, Department of Molecular Biology and \\ Genetics, Aarhus University, Aarhus, DK-8000, Denmark. \\ Correspondence to S.L.-A. or T.H.J. \\ e-mail: sla@mbg.au.dk or thj@mbg.au.dk
}

Abstract: Nonsense-mediated mRNA decay (NMD) is probably the best characterised eukaryotic RNA degradation pathway. Through intricate steps, a set of NMD factors recognise and degrade mRNAs harboring translation termination codons positioned in abnormal contexts. However, NMD is not only part of a general cellular quality control system that prevents the production of aberrant proteins. Indeed, mammalian cells critically depend on NMD to also dynamically adjust their normal transcriptomes and proteomes to a given physiological condition. In this Review we describe how NMD targets mRNAs, the types of mRNAs that are targeted, and discuss the roles of NMD in cellular stress, differentiation and maturation processes.

\section{Introduction}

The ability to regulate gene expression in an organised and adaptable manner is crucial for all life. This is important not only for the execution of cellular processes initiated by stress, cell cycle progression or differentiation, but also for maintaining cellular homeostasis. The expression of a protein-coding gene is controlled by balancing the synthesis, processing, translation and degradation of its corresponding mRNA. Additionally, mature mRNAs must be of a sufficient quality to encode a functional protein. To accomplish this, eukaryotic cells 
employ surveillance systems that scrutinise mRNA quality ${ }^{1,2}$. One such system is the translation-dependent, nonsense-mediated mRNA decay (NMD) pathway, which recognises and eliminates mRNAs with translation termination codons positioned in suboptimal contexts. A typical target has a termination codon positioned more than 50-55 nucleotides upstream of the last exon-exon junction or harbors a long 3' untranslated region (UTR). For historic reasons NMDtriggering translation termination codons are generically termed 'premature termination codons' (PTCs), because of their initial identification in transcripts with truncated open reading frames (ORFs). However, there are now many examples of mRNAs encoding full-length proteins that are nevertheless targeted by NMD ${ }^{3-6}$. Yet, here we will refer, for simplicity, to any NMD-activating codon as a PTC.

Translation termination at a PTC leads to the dynamic assembly and activation of a multiprotein complex. This includes the central ATP-dependent RNA helicase up frameshift 1 (UPF1), which recruits other enzymes responsible for RNA degradation. Increased knowledge about the inner workings of the NMD machinery along with the use of progressively sophisticated experimental methodologies has allowed assessment of its global impact. This points to a farreaching role of NMD in the dynamic regulation of gene expression via its influence on the stability of a wide range of mRNAs. Such regulation is imposed through controlled changes to the coding genomic DNA, pre-mRNA processing or choice of ORF during mRNA translation, which ultimately determines whether the translated mRNA is targeted.

Studies in eukaryotic model organisms such as Saccharomyces cerevisiae, Caenorhabditis elegans and Drosophila melanogaster have been invaluable for the progression of our understanding of NMD. In this Review we primarily focus on mammalian cells while still drawing on accumulated knowledge from other species. After outlining an up-to-date model of how NMD-targeted mRNAs are recognised and eliminated, we proceed to discuss the impact of NMD on gene expression and how NMD-activating features are introduced into mRNAs. Finally, we describe the importance of NMD during differentiation and development as well as in cellular stress responses. 


\section{mRNA-targeting by NMD}

PTC recognition occurs during translation termination and requires several proteins to ultimately degrade the mRNA. In this section we provide an overview of the factor interactions and post-translational modifications that take place during NMD in mammalian cells.

UPF1 - the master regulator of NMD. The proteins UPF1, UPF2 and UPF3 (which has two mammalian paralogues: UPF3A and UPF3B or UPF3X) constitute core NMD factors conserved in all studied eukaryotes ${ }^{3,4,7}$. UPF1 is considered to be the principal NMD factor because it is central to most, if not all, steps from recognition of the PTC-containing mRNAs until their degradation. The ability of UPF1 to selectively target PTC-containing mRNAs depends on its ATPase and helicase activities ${ }^{8-11}$ as well as the phosphorylation of its amino- and carboxyterminal domains ${ }^{12,13}$. Additionally, activation of NMD requires that UPF1 interacts with protein partners on the targeted mRNA. As an example, UPF2 bridges an interaction between UPF1 and UPF3, forming the UPF1-UPF2-UPF3 complex ${ }^{14-16}$. However, there are different branches leading to NMD-activation, and degradation of some mammalian targets can proceed independently of UPF2 and/or UPF3 $3^{17-22}$.

A number of recent studies suggest that UPF1 binds RNA transiently with limited sequence specificity and no apparent discrimination between PTC-containing and regular mRNAs. UPF1 interactions are primarily detected in the 3' UTR of actively translated mRNAs, but can also be observed within ORFs when translation is inhibited, and even on non-coding RNAs ${ }^{11,23-27}$. Its indiscriminate nature illustrates that RNA binding by UPF1 is not sufficient to activate NMD, which presumably requires the specific recruitment of UPF1 to the terminating ribosome $9,28-31$. 
Distinguishing PTCs from regular termination codons. The mechanism by which NMD-activating translation termination is distinguished from regular translation termination remains poorly understood. However, it is clear that both processes depend on the recognition of the termination codon by the translating ribosome in conjunction with its release factors eRF1 and eRF3 (Fig. 1a). By mimicking the shape of a tRNA molecule, eRF1 recognizes any termination codon while in complex with the GTPase eRF3 in its GTP-bound form. Subsequent steps include the hydrolysis of GTP by eRF3, dissociation of eRF3, release of the nascent polypeptide and dissociation of the ribosomal subunits from the mRNA ${ }^{32}$. Prevailing models propose that activation of NMD initially requires the recruitment of UPF1 to the terminating ribosome via a direct interaction with eRF3, which is avoided during efficient translation termination ${ }^{3,4}$.

A number of studies have demonstrated that juxtaposition of a termination codon and the cytoplasmic poly(A) binding protein PABPC prevents NMD ${ }^{19,30,33-}$ 36. Such proximity may allow PABPC to directly interact with eRF3 and preclude UPF1-eRF3 complex formation on the terminating ribosome ${ }^{36-39}$ (Fig. 1a). However, recent reports have shown that the NMD-suppressive effect of PABPC depends critically on its interaction with the translation initiation factor eIF4G, and that eIF4G itself antagonizes NMD when proximal to a termination codon $^{40,41}$. In the context of a mature cytoplasmic mRNA, eIF4G and PABPC associate with the 5' cap and the 3' poly(A)-tail, respectively, allowing the transcript to adopt a closed-loop conformation that stimulates translational initiation and presumably termination ${ }^{42-44}$. Collectively, these data support a model where efficient translation termination is generally stimulated, and NMD evaded, on mRNAs with short 3' UTRs (Fig. 1a). This may also explain why 5'proximal termination codons fail to trigger efficient $\mathrm{NMD}^{45-47}$. Conversely, mRNAs with long 3' UTRs can be targeted by NMD 20,23,25,34,36,48,49, possibly due to increased nonspecific UPF1 binding23-25 and because the spatial distance between the terminating ribosome and the mRNA termini, connected by the PABPC-eIF4G interaction, renders translation termination inefficient. UPF1 could then interact with eRF3 on the terminating ribosome and subsequently recruit additional NMD factors (Fig. 1b). Importantly, however, not all long 3' 
UTRs trigger NMD ${ }^{50}$, suggesting that 3' UTR sequence composition, structure and associated proteins also contribute to fate of the mRNAs.

NMD is stimulated when the termination codon is situated upstream of the splicing-dependent exon junction complex (EJC) deposited approximately 24 nucleotides upstream of most exon-exon junctions ${ }^{51-53}$. A 3' UTR-bound EJC stimulates NMD because it associates with UPF3 and UPF2 promoting the formation of the UPF1-UPF2-UPF3 complex ${ }^{36,54-58}$ (Fig. 1b). EJCs positioned inside ORFs and within the first $\sim 30$ nucleotides of 3' UTRs are believed to dissociate as a consequence of the first (pioneer) round of translation, whereas EJCs in the 3' UTR remain attached to the mRNA. Thus, a termination codon situated more than 50-55 nucleotides upstream of the last exon-exon junction is predicted to be recognised as a PTC 59 .

PTC-induced degradation of mRNA. After the association of UPF1 and eRF3 on the terminating ribosome, a protein complex assembles to activate NMD. The order of events is not well established and may differ on different mRNA substrates. However, there is evidence suggesting the existence of a transient ribosome-associated complex consisting of eRF1-eRF3, UPF1, the ATPdependent RNA helicase DEAH box polypeptide 34 (DHX34) and SMG1C (suppressor with morphological effect on genitalia 1 complex) comprised of the kinase SMG1 and its regulators SMG8 and SMG99,31,60-62. In a process depending on its ATPase activity, DHX34 dissociates DHX34-UPF1-SMG1C from ribosomeassociated eRF1-eRF3 and instead facilitates its association with UPF2-UPF3EJC $^{31}$ (Fig. 1c). This transition may result in translation termination. Moreover, it promotes SMG1-mediated phosphorylation of UPF19,31,60, which possibly stops further translation initiation events ${ }^{63}$ and enables the recruitment of factors responsible for mRNA degradation (Fig. 1c).

Remarkably, mammalian NMD employs up to four different mechanisms to mediate mRNA decay (Fig. 1c): (1) recruitment of the endonuclease SMG6, which catalyses PTC-proximal mRNA cleavage, producing 5' and 3' cleavage fragments degraded by general cellular exonucleases $22,64-69$; (2) recruitment of the SMG5SMG7 heterodimer, which bridges an interaction with the carbon catabolite repressor protein 4 (CCR4)-NOT deadenylase complex thereby shortening the 
poly(A)-tail to stimulate mRNA decapping by the general decapping complex ${ }^{70-}$

72; (3) recruitment of the decapping enhancer proline-rich nuclear receptor coactivator 2 (PNRC2), possibly in a complex with SMG5, which recruits the general decapping complex ${ }^{73-75}$; and/or (4) direct recruitment of the general decapping complex ${ }^{72}$. Decapping is generally followed by decay from the $5^{\prime}$ end by the 5'-3' exonuclease XRN1. It is unknown whether all these events occur simultaneously or according to a certain priority. However, it is likely that PTCpositioning relative to transcript termini as well as the structure of the targeted mRNA determine which decay pathway is kinetically favored.

In addition to mRNA degradation, SMG6 and SMG5-SMG7 may also be required for other steps in NMD. Most notably, the SMG5-SMG7 heterodimer interacts directly with protein phosphatase $2 \mathrm{~A}(\mathrm{PP} 2 \mathrm{~A})$, which is likely responsible for the dephosphorylation and ensuing recycling of UPF113,76,77.

\section{Effects of NMD on gene expression}

Characterisation of the major NMD components has enabled the systematic identification of targeted mRNAs. The ensuing transcriptome-wide analyses of cells with reduced, or disrupted, NMD activity pointed to NMD as an important modulator of gene expression, also targeting mRNAs encoding full-length proteins $18,49,68,69,78-90$. Remarkably, for example, up to $\sim 30 \%$ of all expressed genes in mouse liver cells were found to produce one or more putative NMD targets ${ }^{86}$. Importantly, however, most transcriptome-wide studies have relied on measurements of changes in steady-state mRNA levels after extended periods of reduced NMD activity, and this might have led to misinterpretations of results due to secondary effects. As an example, a number of splicing activators were upregulated in UPF2-deficient mouse liver cells, and these have likely caused non-physiological splicing events that could incorrectly be interpreted as being physiological NMD-substrates ${ }^{86}$. Global identification of NMD targets has also been attempted based on the detection of NMD-specific RNA intermediates or by the direct monitoring of RNA half-lives $68,69,85,87$. This increases the chance of finding direct NMD targets, although the underlying experimental regime still 
relies on prolonged depletion of central NMD factors. Approaches for identification of direct targets of NMD are further discussed in Box 1.

Transcriptome-wide analyses of liver and bone marrow cells from UPF2compromised animals suggested the existence of both ubiquitous and cell typespecific NMD-regulated mRNAs ${ }^{82,83,86}$. Among the former are, for example, subsets of mRNAs derived from pre-mRNAs that harbour one or more small nucleolar RNA (snoRNAs) within their introns. These pre-mRNAs produce, as a consequence of snoRNA biogenesis, non-functional mRNA by-products, which are efficiently purged by NMD ${ }^{69,82,83}$. Furthermore, NMD restricts the expression of several stress-related mRNAs during non-stress conditions ${ }^{85,91-94}$, and NMD is also important for maintaining the homeostasis of specific gene expression networks such as those encoding splicing activators and repressors $80,81,86,95$ as well as components of the NMD pathway itself ${ }^{20,49}$. Finally, several long noncoding RNAs (lncRNAs) are targeted by NMD because they encode small ORFs ${ }^{6}$. A subset of such lncRNAs may in fact encode functional micro-peptides and therefore be wrongly annotated as non-coding. Others, such as growth arrestspecific 5 (GAS5) and ZNFX1 antisense RNA 1 (ZFAS1), can be considered as IncRNA-mRNA hybrids, because they are functional lncRNAs that also undergo translation ${ }^{96-98}$. It is an interesting possibility that some functional non-coding RNAs may engage the translation machinery with the sole purpose of achieving efficient degradation by NMD.

NMD-controlled mRNAs are diverse. Some encode full-length proteins while others have truncated $\mathrm{ORFs}^{49,78,86}$. Structural and biochemical evidence suggest that UPF1 has ubiquitin-ligase activity ${ }^{99,100}$, which may stimulate the proteasome to degrade aberrant proteins encoded by PTC-containing mRNAs ${ }^{101,102}$. The data supporting such NMD-associated protein degradation is limited to a few model substrates in S. cerevisiae and it appears that UPF1-stimulated acceleration of degradation only occurs for proteins that are inherently unstable ${ }^{101,102}$. Hence, NMD-targeted mRNAs probably give rise to protein products, when these are intrinsically stable. Moreover, the penetrance of NMD varies between PTCcontaining mRNAs, which is reflected by observations suggesting that NMD targets can be degraded not only during their pioneering translation event ${ }^{103}$, but also during subsequent rounds of translation ${ }^{23,104,105}$. In summary, NMD 
targets a wide variety of mRNAs with variable outcomes at both transcript and protein levels.

\section{Types of NMD-targeted mRNAs}

Although the core process of NMD is executed in the cytoplasm during translation ${ }^{106}$, the susceptibility of mRNAs to NMD is also determined by nuclear processes. As an example, the EJC is loaded onto spliced mRNAs prior to their nuclear export ${ }^{54}$. UPF1, UPF2 and UPF3 themselves shuttle between the nucleus and cytoplasm, and at least UPF3 may already associate with mRNAs via the EJC in the nucleus ${ }^{14,54}$. NMD-targets can conceptually be divided into at least three, partially overlapping, categories based on where in the gene expression circuit the defining NMD-triggering feature is introduced: (1) random DNA mutations or programmed genomic rearrangements can generate PTCs; (2) alternative premRNA synthesis or processing can generate PTC-containing isoforms; and (3) translation of alternative PTC-containing ORFs embedded within mature mRNAs that encode full-length proteins. Below we discuss in more detail these three categories of NMD-targeted transcripts. 
Random mutations and programmed genomic rearrangements. PTCs can arise via nonsense or frameshift mutations in the coding DNA (Suppl. Fig. S1a, panels 2-3). Mutations inflicting errors in splicing or translation start site may also introduce PTCs (Suppl. Fig. S1a, panels 4-6). Such PTC-introducing events constitute approximately $30 \%$ of all known disease-associated mutations ${ }^{107}$. NMD targets transcripts produced from such irreversibly altered alleles, essentially generating a loss-of-function phenotype. In this way, cells are protected against the potentially dominant negative activity of truncated proteins (Fig. 2). Thus, NMD can be beneficial for heterozygous carriers of PTC-generating mutations if expression from the healthy allele is sufficient to maintain a near-normal function of the affected gene. This is the case for a version of the blood disorder $\beta$-thalassemia caused by PTC-introducing mutations in the hemoglobin, beta (HBB or $\beta$-globin) gene. However, NMD-triggered loss-of-function may also cause disease due to haploinsufficiency (Fig. 2) as exemplified by PTC-generating mutations in the SRY (sex determining region Y)-box 10 (SOX10) gene, which lead to Waardenburg syndrome type $4 \mathrm{C}^{108,109}$.

While PTCs can be introduced randomly through DNA mutations, they are systematically generated as a consequence of the programmed mutagenesis of the vertebrate T-cell receptor (TCR) and immunoglobulin (Ig) genes. This process, that takes place during maturation of the immune system in lymphocytes, serves to generate immune receptor diversity. TCR and $I g$ genes are subjected to programmed rearrangements where specific DNA elements are joined by recombination during maturation of T- and B-cells, respectively. As part of this process, short stretches of nucleotides are randomly trimmed away or introduced at the junctions between the joined DNA elements ${ }^{110}$. This changes the reading frame in an expected two-thirds of the cases, resulting in PTCs. Additionally, Ig genes undergo somatic hypermutation within a specific region in antigen-stimulated B-cells, which may also generate PTCs in the $I g$ mRNAs$^{110}$. Such PTC-containing mRNAs, encoding non-functional immune receptors, are presumably degraded by NMD 82,111 . The $T C R-\beta$ loci are sequentially rearranged during T-cell maturation, and if the first event generates a functional TCR- $\beta$ allele, T-cell maturation ensues without the second rearrangement (Fig. 3). In case the first rearrangement produces a PTC, the second allele is rearranged. T- 
cell maturation only continues if this second event is successful and the original PTC-containing Tcr- $\beta$ mRNA is effectively eliminated by NMD in mouse T-cells ${ }^{82}$ (Fig. 3). In mice with T-cell lineage-specific disruption of UPF2 expression, the population of resting T-cells is considerably diminished compared to control animals ${ }^{82}$. Additionally, the surviving cells almost exclusively express mRNAs derived from productively rearranged $T c r-\beta$ loci, suggesting that T-cells with one functional and one PTC-containing Tcr- $\beta$ allele fail to survive in the absence of $\mathrm{NMD}^{82}$ (Fig. 3). This implies that the clearance of PTC-containing Tcr- $\beta$ mRNAs is vital for maturation of sufficient populations of T-cells.

mRNA isoforms. A gene can encode different transcript isoforms due to the alternative use of transcription initiation sites as well as the alternative splicing or 3' end processing of its pre-mRNA. In humans it is estimated that most introncontaining genes can produce at least two alternative transcript isoforms ${ }^{112}$, and in a substantial number of cases at least one of these is predicted to trigger NMD $^{113}$. Sensitivity to NMD can be conveyed by: (1) inclusion of an alternative exon within the ORF, which may contain a PTC or introduce one by causing a frameshift; (2) exclusion of an exon from the ORF leading to frameshifting; and (3) splicing within the pre-mRNA 3' UTR resulting in the assembly of an EJC downstream of a regular termination codon functionally changing it to a PTC78,80,86,95 (Suppl. Fig. S1b). In addition, alternative transcription initiation and 3' end processing may produce NMD-prone mRNA due to the introduction of an alternative initiation codon ${ }^{114}$ or by extending the 3' UTR, respectively (Suppl. Fig. 1c).

Although PTC-containing mRNAs can arise fortuitously by errors in transcription or pre-mRNA processing, leading to their removal by NMD as part of RNA quality control, it is evident that alternative splicing combined with NMD (AS-NMD) is also associated with the regulation of normal gene expression. This is because several alternatively spliced mRNA isoforms with predicted PTCs accumulate following the disrupted expression of NMD factors $49,69,78,80,81,84,86,95,115$. For example, genes encoding the serine/arginine-rich splicing factor (SRSF) splicing activators and the heterogeneous nuclear ribonucleoprotein (HNRNP) splicing repressors are subjected to AS-NMD. The NMD-targeted mRNAs originating from 
these genes are alternatively spliced in a manner that corresponds to the function of the encoded protein, that is, PTCs are introduced into SRSF and HNRNP mRNA isoforms by exon inclusion and exon exclusion, respectively $80,81,95$ (Fig. 4). Besides this striking correlation, a subset of specific factors from both classes of splicing regulators were demonstrated to control the NMD-triggering splicing events of their own transcripts ${ }^{116,117}$. Additionally, disrupted UPF2 function in mouse liver triggers the accumulation of a number of SRSF splicing activators concurrent with the appearance of a sizeable number of abnormal splice variants from unrelated genes ${ }^{86}$. Collectively, these data support a model in which these families of splicing factors keep their own expression levels under control by causing AS-NMD of their own transcripts. Such homeostasis of splicing factor expression in turn controls global alternative splicing in cells ${ }^{80,81,86,95}$ (Fig. 4).

Intron retention, a type of alternative splicing, has been the focus of much attention recently ${ }^{112}$. In contrast to other alternative splicing events, transcripts with retained introns are difficult to distinguish from unspliced pre-mRNAs. Still, recent studies demonstrated that many genes produce mRNAs with retained introns during differentiation processes ${ }^{90,118}$. A transcriptome-wide comparison of different stages in mouse granulocyte development identified 121 retained introns originating from 86 genes; the retention of most introns increased during the differentiation process. The majority of these intron retention events predictably introduce PTCs and at least one-fourth of these are likely substrates of NMD, based on detectable mRNA accumulation upon inhibition of NMD ${ }^{90}$. Although indications exist that these intron retention events are important for proper granulocyte development, it is not clear whether this depends on NMD perse. 
Alternative translation. Approximately $40 \%$ of all mammalian mRNAs contain putative upstream ORFs (uORFs) with possible regulatory impact via the propensity to trigger NMD owing to frameshift-induced PTCs ${ }^{119}$. Indeed, many mRNAs that accumulate upon NMD factor depletion contain uORFs $25,49,78,120$ and their degrees of translation correlate well with their estimated sensitivities to NMD as measured by ribosome profiling and RNA levels upon inhibition of NMD, respectively ${ }^{25}$. The conditions under which translation is reprogrammed to favor the use of non-functional uORFs relative to functional ORFs are largely elusive but likely to be mRNA-specific. The mRNA encoding activating transcription factor 4 (ATF4), a central transcription factor in the integrated stress response, is a well-studied example. Although the exact mechanism is unknown, it has been suggested that the initial translation of a three-codon long uORF is followed by translation re-initiation at either a second, non-functional UORF or at the regular, protein-coding ORF. Translation of the protein-coding ORF is favored during stress conditions, whereas the second $\mathrm{UORF}$, which is NMD-sensitive, is preferentially used in normal conditions ${ }^{91,121}$ (Fig. 5a).

Programmed ribosomal frameshifting (PRF) is another potentially prevalent trigger of NMD. Up to $10 \%$ of eukaryotic genes are predicted to encode RNA elements that induce PRF by one nucleotide in the 5'-direction (-1 PRF). The majority of these lead to premature translation termination ${ }^{122}$. A recent study described an NMD-inducing -1 PRF in a mRNA encoding the cytokine receptor CCR5. Here, the PRF is stimulated by specific microRNAs (miRNAs) (Fig. 5b) and a similar mechanism was implied for six other mRNAs encoding cytokine receptor subunits ${ }^{123}$.

An example of a less common alternative translation process that can trigger NMD is provided by mRNAs encoding selenoproteins ${ }^{124}$. Common for these mRNAs ( $\sim 25$ in humans) is the presence of a selenocysteine insertion sequence (SECIS) in their 3' UTRs, which, when selenium is available, allows the recruitment of a specialised translational elongation factor bound to selenocysteine-tRNA. This facilitates the insertion of a selenocysteine at one or more UGA termination codons ${ }^{124}$. Selenium is an essential micronutrient and a subset of selenoproteins play central roles in controlling cellular redox status. There are data supporting the existence of a hierarchy among selenoproteins 
where production of some is favored over others when selenium is scarce. This presumably serves to ensure the preferential expression of the most important selenoproteins in a given tissue ${ }^{125}$. NMD may, at least in part, play a role in establishing such a hierarchy, as reductions in selenoprotein mRNA levels match reduced selenoprotein levels upon selenium deficiency ${ }^{125}$. Specifically, based on their position relative to exon-exon junctions many of these UGA codons are potential PTCs and so about half of the selenoprotein mRNAs are predicted to be targeted by NMD in the absence of selenium, whereas the incorporation of selenocysteine instead is expected to result in productive translation ${ }^{126}$ (Fig. 5c). While some selenoprotein mRNAs were demonstrated to be sensitive to depletion of NMD factors ${ }^{126,127}$ and display reduced half-lives in low selenium concentrations $^{126}$, it still remains to be established whether NMD is critical for modulating the cellular response to reduced selenium levels.

\section{Physiological functions of NMD}

The efficiency of NMD can be regulated in response to various cues. This can be elicited by modulation of the overall activity of the NMD pathway or at the level of individual subsets of transcripts, whose NMD-susceptibility can be specifically altered via processes that control alternative mRNA biogenesis or translation. The ability of cells to adjust the global and/or transcript-specific activity of NMD is critical for a number of physiological processes.

NMD-factors are essential for embryonic development. There are several studies implying that NMD factors are important for development. Strongest evidence comes from disrupted expression of UPF1, UPF2, SMG1 or SMG6 in mice, which all die at an early embryonic stage ${ }^{82,84,128,129}$. However, since some NMD factors are also involved in cellular pathways that are distinct from NMD, these observations are not sufficient to conclude that NMD per se is essential for mammalian embryonic development. For example, all the mentioned factors also take part in telomere maintenance and UPF1 is a central component in histone mRNA degradation and Staufen1-mediated mRNA decay (SMD) ${ }^{130,131}$. Although the mechanisms of the latter two pathways have not been determined in detail, it has been established that they do not depend on UPF2 ${ }^{132,133}$. Thus, mouse 
embryonic lethality caused by disrupted UPF2 expression can likely be ascribed to NMD and/or telomere maintenance. The distinct roles of NMD and telomere maintenance during developmental processes were addressed in a recent study of mice using conditionally disrupted SMG6 expression ${ }^{129}$. First, it was established that SMG6-deficient embryonic stem cells (ESCs) proliferate, but fail to differentiate, which provides a plausible explanation for the observed embryonic lethality. Next, variants of the SMG6 protein lacking specific domains necessary for NMD or for telomere maintenance were re-introduced to ESCs lacking endogenous SMG6. This demonstrated that NMD-related domains are required whereas telomere maintenance is dispensable for differentiation ${ }^{129}$. However, although there is presently no evidence for SMG6 being involved in SMD or histone mRNA decay, it cannot be excluded that these pathways could also be involved. The differentiation defect in SMG6-depleted ESCs could be attributed to a robust increase in the levels of the transcription factor c-Myc and its cognate mRNA. Yet, it is not clear whether $c-M y c$ mRNA is a direct substrate for NMD, since a reporter mRNA including the 3' UTR of $c-M y c$ only accumulated slightly in response to disrupted SMG6 expression ${ }^{129}$. Taken together, although not firmly proven, the described observations suggest that NMD is crucial for embryonic development. 
The potency of NMD affects cell type diversification. NMD has been implicated as an important player in cellular proliferation, differentiation and maturation programs. As an example, detailed analyses using conditional, tissue-specific disruption of UPF2 expression in mice revealed that it is critical for the maintenance of hematopoietic stem and progenitor cells, the maturation of Tcells, the activation of resting lymphocytes ${ }^{82}$, fetal liver development as well as the homeostasis and regeneration of the adult liver ${ }^{83}$. Moreover, different types of stem- or progenitor cells appear to have variable requirements for NMD activity. Proliferation and maintenance of the stem cell state is supported and differentiation is inhibited in mouse ESCs depleted of SMG1, SMG5, SMG6, UPF1 or UPF ${ }^{129}$. In contrast, disruption of UPF2 expression prevents the proliferation of hematopoietic stem and progenitor cells ${ }^{82}$. Additionally, there are contrasting observations for neuronal stem cells, where UPF3B ${ }^{134}$ and UPF1135 depletion prevents and supports differentiation (and supports and prevents proliferation), respectively. However, the role of NMD is supposedly more complex than can be deduced from simple depletion experiments, as indicated by the fine-tuned downregulation of NMD activity during the differentiation of myoblasts to myotubes ${ }^{133}$.

A recent study applied a more refined approach to study the role of NMD in neuronal stem cells and their differentiation into neurons ${ }^{135}$. Here, a decrease in the levels of mRNAs encoding UPF1, UPF3B and other central NMD factors was observed during in vitro neural differentiation of mouse neuronal stem-like cells. This was accompanied by a decrease in NMD activity as measured by a reportermRNA system. By measuring levels of markers of neural differentiation and the stem-cell state as well as markers for cellular proliferation, it was deduced that (1) modest overexpression of UPF1 in neuronal stem-like cells treated with a differentiation-inducing drug inhibited differentiation and instead upheld a stem-like proliferative state; (2) depletion of UPF1 in undifferentiated neuronal stem-like cells decreased their proliferation and induced neural differentiation and maturation; and (3) isolated neuronal stem cells from UPF3B-deficient mice displayed increased levels of differentiation markers. It is therefore likely that high NMD activity in undifferentiated neuronal stem cells maintains their proliferative stem-like state, whereas reduction of NMD activity is critical for 
their differentiation into neurons ${ }^{135}$ (Fig. 6a). Further experiments revealed that subsets of mRNAs encoding proliferation inhibitors and neural differentiation factors are direct targets of NMD, which may explain why modulation of NMD activity can change the cell state. Downregulation of levels of UPF1, UPF3B and possibly other NMD factors during neuronal differentiation is controlled, at least in part, by a set of miRNAs that are upregulated in response to neuronal differentiation signals ${ }^{135,136}$. This regulatory circuit appears to be self-sustaining, since these miRNAs suppress NMD activity, and in turn high NMD activity indirectly represses the expression of the miRNAs ${ }^{135}$ (Fig. 6a).

Local modulation of NMD during neural development. Besides its role in differentiation of neurons from stem cell precursors, NMD also plays a role at later stages of neural development $88,115,134$. A recent study demonstrated that NMD factors are enriched within the axonal growth cones of neurons ${ }^{115}$ (Fig. 6b). This restricts the expression of an alternative splice variant of the Roundabout homolog 3 (Robo3) gene, which contains a PTC in a retained intron. The splice variant is translated locally only when commissural neurons pass the spinal cord midline during neural development (Fig. 6b). The resulting R0B03.2 protein isoform is a receptor important for guiding the trajectory of the axon after passage of the midline ${ }^{137}$. Failure of NMD to degrade Robo3.2 mRNA in UPF2 depleted mouse commissural neurons leads to increased ROB03.2 receptor levels in the growth cones and erroneous migration of the axons after passage of the midline ${ }^{115}$. This suggests that NMD is employed to degrade the Robo3.2 mRNA after one or a few rounds of translation, to ensure that the expression of ROB03.2 protein is tightly restricted temporally and quantitatively ${ }^{115}$. This phenomenon raises the possibility that other genes utilize a similar strategy to control their local protein levels and local enrichment or depletion of NMD factors may also be important in other cell types.

The importance of NMD during neural development is consistent with the finding that mutations in the human $X$-linked $U P F 3 B$ gene cause various degrees of intellectual disability ${ }^{138}$, which is accompanied by deregulation of mRNAs encoding proteins with functions related to neuronal processes ${ }^{88}$. Furthermore, copy-number variations of other genes encoding factors involved in NMD, 
including the heterozygous deletion of the UPF2 gene, are frequent in individuals with intellectual disability and/or other neuro-developmental disorders ${ }^{89}$.

NMD modulates, and is modulated by, cellular stress. Depletion of NMD factors has consistently resulted in increased levels of mRNAs encoding stress-related proteins $68,69,78,82,83,85,87$. This could happen because NMD targets stress-related mRNAs, and/or because the downregulation of NMD induces stress. While there is evidence both for ${ }^{93}$ and against ${ }^{82,94}$ the latter, it is nonetheless clear that many stress-related mRNAs are NMD targets in unstressed cells, but escape degradation during stresses such as the unfolded protein response (UPR) and oxygen deprivation ${ }^{85,91-94}$. Many of these NMD-sensitive transcripts contain uORFs or long 3' UTRs as their putative NMD-activating features ${ }^{94}$.

One example of a stress response is the UPR, which is induced by the accumulation of unfolded or misfolded proteins in the endoplasmic reticulum (ER). It was recently demonstrated that depletion of the non-essential NMD factor UPF3B renders both cultured human cells and whole mice highly sensitive to mild ER stresses that would normally not induce the UPR ${ }^{94}$. Upregulation of the PTC-containing mRNA encoding the functional protein inositol requiring enzyme $1 \alpha$ (IRE1 $\alpha)$, a central sensor in the UPR, may underlie such UPR sensitization. This is supported by three lines of evidence: (1) IRE1 $\alpha$ protein levels increased in UPF3B depleted cells; (2) partial depletion of IRE1 $\alpha$ (back to normal levels) desensitised UPF3B depleted cells; and (3) IRE1 $\alpha$ overexpression recapitulated the phenotype of UPF3B depleted cells ${ }^{94}$. Moreover, half-life analyses following depletion of NMD factors suggested that the Ire $1 \alpha$ mRNA is a direct target of the NMD pathway. It was therefore suggested that NMD serves to restrict IRE1 $\alpha$ levels to avoid unwarranted initiation of the UPR in response to mild ER stress ${ }^{94}$. Interestingly, the sensitivity to NMD of UPR-effector mRNAs, including Atf4, decreases during the UPR to allow sufficient production of proteins to muster a robust response ${ }^{93,94}$. When the UPR has run its course, NMD is again needed to dampen the levels of UPR-related factors, as was indicated by the inefficient termination of UPR in human cells and mice depleted for UPF $3 B^{94}$ (Fig. 6c). 
The UPR and many other stress-activated signalling pathways reduce global translation while allowing unchanged, or even increased, translation of stressrelated mRNAs ${ }^{139}$ (Fig. 6c). Importantly, the global reduction of translation likely causes the bulk of non-stress-responsive, PTC-containing mRNAs to escape translation and therefore also to evade $\mathrm{NMD}^{85,91,93,94,140 .}$ Stress-responsive mRNAs likely have specific mechanisms to ensure their continued translation and escape from NMD during stress. For example, ATF4 undergoes reprogramming from translating a uORF to the functional ORF, which allows both stabilization of the mRNA and production of functional ATF4 protein ${ }^{85,91,121}$ (Fig. 5a). A general reduction in NMD activity on translated mRNAs during stress responses has also been suggested ${ }^{85}$. This could possibly happen by sequestration of NMD factors in stress granules as observed for UPF1 and SMG191,141 or if levels of rate-limiting NMD factors are reduced due to the overall reduction of normal translation.

\section{Concluding remarks}

The NMD pathway has two established functions in mammalian cells. Firstly, it degrades mRNAs encoding truncated proteins with no or undesired functions. In this capacity, it prevents the accumulation of potentially harmful polypeptides. Whether this quality control measure is generally essential for cellular function and survival has not been rigorously established. Yet, in the specialized case of Tcell maturation, it is highly likely that NMD-facilitated removal of mRNAs encoding non-functional, truncated TCR- $\beta$ receptors is essential for generation of normal levels of mature T-cells ${ }^{82}$. Secondly, by targeting a subset of mRNAs with intact ORFs, NMD is a direct modulator of the expression of functional proteins. The sensitivity of an mRNA to NMD can be altered as illustrated by the translation of different ORFs in the ATF4 mRNA under normal or stress conditions ${ }^{91,121}$. Additionally, regulation of expression levels and localization of NMD factors can modulate the activity of the NMD pathway. These mechanisms may be important for the control of protein levels during a variety of cellular processes. Presently, there is evidence supporting a critical role of NMD when 
cells sense stress and execute stress responses ${ }^{85,91-94}$ and during differentiation and developmental processes $82,83,115,129,134,135$.

Many unresolved questions remain. A detailed understanding of the mechanism of target recognition and degradation is pivotal to enable experimental designs that address the cellular functions of NMD. Increasingly sophisticated methods such as genome editing tools and tailored next generation sequencing approaches may allow the study of NMD function less invasively and thus more directly than previously possible. Notwithstanding the expected improvement in our understanding of NMD, it is already evident that this system is a multifaceted regulatory tool of the eukaryotic cell.

\section{References}

1. Lykke-Andersen, J. \& Bennett, E. J. Protecting the proteome: Eukaryotic cotranslational quality control pathways. J. Cell Biol. 204, 467-476 (2014).

2. Muhlemann, 0. \& Jensen, T. H. mRNP quality control goes regulatory. Trends Genet. 28, 70-77 (2012).

3. Kervestin, S. \& Jacobson, A. NMD: a multifaceted response to premature translational termination. Nat. Rev. Mol. Cell Biol. 13, 700-712 (2012).

4. Schweingruber, C., Rufener, S. C., Zund, D., Yamashita, A. \& Muhlemann, 0. Nonsense-mediated mRNA decay - mechanisms of substrate mRNA recognition and degradation in mammalian cells. Biochim. Biophys. Acta 1829, 612-623 (2013).

5. Popp, M. W. \& Maquat, L. E. Organizing principles of mammalian nonsensemediated mRNA decay. Annu. Rev. Genet. 47, 139-165 (2013).

6. Smith, J. E. \& Baker, K. E. Nonsense-mediated RNA decay - a switch and dial for regulating gene expression. Bioessays 37, 612-623 (2015).

7. Siwaszek, A., Ukleja, M. \& Dziembowski, A. Proteins involved in the degradation of cytoplasmic mRNA in the major eukaryotic model systems. RNA Biol. 11, 1122-1136 (2014).

8. Weng, Y., Czaplinski, K. \& Peltz, S. W. Genetic and biochemical characterization of mutations in the ATPase and helicase regions of the Upf1 protein. Mol. Cell. Biol. 16, 5477-5490 (1996).

9. Kashima, I. et al. Binding of a novel SMG-1-Upf1-eRF1-eRF3 complex (SURF) to the exon junction complex triggers Upf1 phosphorylation and nonsensemediated mRNA decay. Genes Dev. 20, 355-367 (2006). 
10. Franks, T. M., Singh, G. \& Lykke-Andersen, J. Upf1 ATPase-dependent mRNP disassembly is required for completion of nonsense- mediated mRNA decay. Cell 143, 938-950 (2010).

11. Kurosaki, T. et al. A post-translational regulatory switch on UPF1 controls targeted mRNA degradation. Genes Dev. 28, 1900-1916 (2014).

12. Yamashita, A., Ohnishi, T., Kashima, I., Taya, Y. \& Ohno, S. Human SMG-1, a novel phosphatidylinositol 3-kinase-related protein kinase, associates with components of the mRNA surveillance complex and is involved in the regulation of nonsense-mediated mRNA decay. Genes Dev. 15, 2215-2228 (2001).

13. Ohnishi, T. et al. Phosphorylation of hUPF1 induces formation of mRNA surveillance complexes containing hSMG-5 and hSMG-7. Mol. Cell 12, 1187-1200 (2003).

14. Lykke-Andersen, J., Shu, M. D. \& Steitz, J. A. Human Upf proteins target an mRNA for nonsense-mediated decay when bound downstream of a termination codon. Cell 103, 1121-1131 (2000).

15. Serin, G., Gersappe, A., Black, J. D., Aronoff, R. \& Maquat, L. E. Identification and characterization of human orthologues to Saccharomyces cerevisiae Upf2 protein and Upf3 protein (Caenorhabditis elegans SMG-4). Mol. Cell. Biol. 21, 209-223 (2001).

16. Melero, R. et al. The cryo-EM structure of the UPF-EJC complex shows UPF1 poised toward the RNA 3' end. Nat. Struct. Mol. Biol. 19, 498-505, S1-2 (2012).

17. Gehring, N. H. et al. Exon-junction complex components specify distinct routes of nonsense-mediated mRNA decay with differential cofactor requirements. Mol. Cell 20, 65-75 (2005).

18. Chan, W. K. et al. An alternative branch of the nonsense-mediated decay pathway. EMBO J. 26, 1820-1830 (2007).

19. Ivanov, P. V., Gehring, N. H., Kunz, J. B., Hentze, M. W. \& Kulozik, A. E. Interactions between UPF1, eRFs, PABP and the exon junction complex suggest an integrated model for mammalian NMD pathways. EMBO J. 27, 736-747 (2008).

20. Huang, L. et al. RNA homeostasis governed by cell type-specific and branched feedback loops acting on NMD. Mol. Cell 43, 950-961 (2011).

21. Metze, S., Herzog, V. A., Ruepp, M. D. \& Muhlemann, O. Comparison of EJCenhanced and EJC-independent NMD in human cells reveals two partially redundant degradation pathways. RNA 19, 1432-1448 (2013).

22. Boehm, V., Haberman, N., Ottens, F., Ule, J. \& Gehring, N. H. 3' UTR length and messenger ribonucleoprotein composition determine endocleavage efficiencies at termination codons. Cell. Rep. 9, 555-568 (2014).

23. Hogg, J. R. \& Goff, S. P. Upf1 senses 3'UTR length to potentiate mRNA decay. Cell 143, 379-389 (2010).

24. Kurosaki, T. \& Maquat, L. E. Rules that govern UPF1 binding to mRNA 3' UTRs. Proc. Natl. Acad. Sci. U. S. A. 110, 3357-3362 (2013). 
25. Hurt, J. A., Robertson, A. D. \& Burge, C. B. Global analyses of UPF1 binding and function reveal expanded scope of nonsense-mediated mRNA decay. Genome Res. 23, 1636-1650 (2013).

26. Zund, D., Gruber, A. R., Zavolan, M. \& Muhlemann, O. Translation-dependent displacement of UPF1 from coding sequences causes its enrichment in 3' UTRs. Nat. Struct. Mol. Biol. 20, 936-943 (2013).

27. Gregersen, L. H. et al. MOV10 Is a 5' to 3' RNA helicase contributing to UPF1 mRNA target degradation by translocation along 3' UTRs. Mol. Cell 54, 573-585 (2014).

References 11 and 23-27 suggest that UPF1 binding of RNA is non-specific and does not discriminate NMD from non-NMD substrates, but becomes specific following NMD activation.

28. Czaplinski, K. et al. The surveillance complex interacts with the translation release factors to enhance termination and degrade aberrant mRNAs. Genes Dev. 12, 1665-1677 (1998).

29. Wang, W., Czaplinski, K., Rao, Y. \& Peltz, S. W. The role of Upf proteins in modulating the translation read-through of nonsense-containing transcripts. EMBO J. 20, 880-890 (2001).

30. Amrani, N. et al. A faux 3'-UTR promotes aberrant termination and triggers nonsense-mediated mRNA decay. Nature 432, 112-118 (2004).

31. Hug, N. \& Caceres, J. F. The RNA helicase DHX34 activates NMD by promoting a transition from the surveillance to the decay-inducing complex. Cell. Rep. 8, 1845-1856 (2014).

32. Dever, T. E. \& Green, R. The elongation, termination, and recycling phases of translation in eukaryotes. Cold Spring Harb Perspect. Biol. 4, a013706 (2012).

33. Behm-Ansmant, I., Gatfield, D., Rehwinkel, J., Hilgers, V. \& Izaurralde, E. A conserved role for cytoplasmic poly(A)-binding protein 1 (PABPC1) in nonsensemediated mRNA decay. EMBO J. 26, 1591-1601 (2007).

34. Eberle, A. B., Stalder, L., Mathys, H., Orozco, R. Z. \& Muhlemann, O. Posttranscriptional gene regulation by spatial rearrangement of the 3 ' untranslated region. PLoS Biol. 6, e92 (2008).

35. Silva, A. L., Ribeiro, P., Inacio, A., Liebhaber, S. A. \& Romao, L. Proximity of the poly(A)-binding protein to a premature termination codon inhibits mammalian nonsense-mediated mRNA decay. RNA 14, 563-576 (2008).

36. Singh, G., Rebbapragada, I. \& Lykke-Andersen, J. A competition between stimulators and antagonists of Upf complex recruitment governs human nonsense-mediated mRNA decay. PLoS Biol. 6, e111 (2008).

37. Hoshino, S., Imai, M., Kobayashi, T., Uchida, N. \& Katada, T. The eukaryotic polypeptide chain releasing factor (eRF3/GSPT) carrying the translation termination signal to the 3 '-Poly(A) tail of mRNA. Direct association of erf3/GSPT with polyadenylate-binding protein. J. Biol. Chem. 274, 16677-16680 (1999). 
38. Cosson, B. et al. Poly(A)-binding protein acts in translation termination via eukaryotic release factor 3 interaction and does not influence [PSI $(+)]$ propagation. Mol. Cell. Biol. 22, 3301-3315 (2002).

39. Kozlov, G. \& Gehring, K. Molecular basis of eRF3 recognition by the MLLE domain of poly(A)-binding protein. PLoS One 5, e10169 (2010).

40. Joncourt, R., Eberle, A. B., Rufener, S. C. \& Muhlemann, O. Eukaryotic initiation factor 4G suppresses nonsense-mediated mRNA decay by two genetically separable mechanisms. PLoS One 9, e104391 (2014).

41. Fatscher, T., Boehm, V., Weiche, B. \& Gehring, N. H. The interaction of cytoplasmic poly(A)-binding protein with eukaryotic initiation factor 4G suppresses nonsense-mediated mRNA decay. RNA 20, 1579-1592 (2014).

References 40 and 41 demonstrate that the ability of PTC-proximal PABPC to render a transcript resistant to NMD depends on its interaction with eIF4G.

42. Wells, S. E., Hillner, P. E., Vale, R. D. \& Sachs, A. B. Circularization of mRNA by eukaryotic translation initiation factors. Mol. Cell 2, 135-140 (1998).

43. Kahvejian, A., Svitkin, Y. V., Sukarieh, R., M'Boutchou, M. N. \& Sonenberg, N. Mammalian poly(A)-binding protein is a eukaryotic translation initiation factor, which acts via multiple mechanisms. Genes Dev. 19, 104-113 (2005).

44. Amrani, N., Ghosh, S., Mangus, D. A. \& Jacobson, A. Translation factors promote the formation of two states of the closed-loop mRNP. Nature 453, 12761280 (2008).

45. Inacio, A. et al. Nonsense mutations in close proximity to the initiation codon fail to trigger full nonsense-mediated mRNA decay. J. Biol. Chem. 279, 3217032180 (2004).

46. Neu-Yilik, G. et al. Mechanism of escape from nonsense-mediated mRNA decay of human beta-globin transcripts with nonsense mutations in the first exon. RNA 17, 843-854 (2011).

47. Peixeiro, I. et al. Interaction of PABPC1 with the translation initiation complex is critical to the NMD resistance of AUG-proximal nonsense mutations. Nucleic Acids Res. 40, 1160-1173 (2012).

This reference provides evidence that the interaction between PABPC and eIF4G is critical to render a mRNA containing a termination codon close to the start codon resistant to NMD.

48. Buhler, M., Steiner, S., Mohn, F., Paillusson, A. \& Muhlemann, O. EJCindependent degradation of nonsense immunoglobulin-mu mRNA depends on 3' UTR length. Nat. Struct. Mol. Biol. 13, 462-464 (2006).

49. Yepiskoposyan, H., Aeschimann, F., Nilsson, D., Okoniewski, M. \& Muhlemann, 0 . Autoregulation of the nonsense-mediated mRNA decay pathway in human cells. $R N A$ 17, 2108-2118 (2011).

50. Toma, K. G., Rebbapragada, I., Durand, S. \& Lykke-Andersen, J. Identification of elements in human long 3' UTRs that inhibit nonsense-mediated decay. RNA 21, 887-897 (2015). 
51. Le Hir, H., Izaurralde, E., Maquat, L. E. \& Moore, M. J. The spliceosome deposits multiple proteins 20-24 nucleotides upstream of mRNA exon-exon junctions. EMBO J. 19, 6860-6869 (2000).

52. Singh, G. et al. The cellular EJC interactome reveals higher-order mRNP structure and an EJC-SR protein nexus. Cell 151, 750-764 (2012).

53. Sauliere, J. et al. CLIP-seq of eIF4AIII reveals transcriptome-wide mapping of the human exon junction complex. Nat. Struct. Mol. Biol. 19, 1124-1131 (2012).

54. Le Hir, H., Gatfield, D., Izaurralde, E. \& Moore, M. J. The exon-exon junction complex provides a binding platform for factors involved in mRNA export and nonsense-mediated mRNA decay. EMBO J. 20, 4987-4997 (2001).

55. Kim, V. N., Kataoka, N. \& Dreyfuss, G. Role of the nonsense-mediated decay factor hUpf3 in the splicing-dependent exon-exon junction complex. Science 293, 1832-1836 (2001).

56. Lykke-Andersen, J., Shu, M. D. \& Steitz, J. A. Communication of the position of exon-exon junctions to the mRNA surveillance machinery by the protein RNPS1. Science 293, 1836-1839 (2001).

57. Gehring, N. H., Neu-Yilik, G., Schell, T., Hentze, M. W. \& Kulozik, A. E. Y14 and hUpf3b form an NMD-activating complex. Mol. Cell 11, 939-949 (2003).

58. Gehring, N. H. et al. Exon-junction complex components specify distinct routes of nonsense-mediated mRNA decay with differential cofactor requirements. Mol. Cell 20, 65-75 (2005).

59. Nagy, E. \& Maquat, L. E. A rule for termination-codon position within introncontaining genes: when nonsense affects RNA abundance. Trends Biochem. Sci. 23, 198-199 (1998).

60. Yamashita, A. et al. SMG-8 and SMG-9, two novel subunits of the SMG-1 complex, regulate remodeling of the mRNA surveillance complex during nonsense-mediated mRNA decay. Genes Dev. 23, 1091-1105 (2009).

61. Arias-Palomo, E. et al. The nonsense-mediated mRNA decay SMG-1 kinase is regulated by large-scale conformational changes controlled by SMG-8. Genes Dev. 25, 153-164 (2011).

62. Melero, R. et al. Structures of SMG1-UPFs complexes: SMG1 contributes to regulate UPF2-dependent activation of UPF1 in NMD. Structure 22, 1105-1119 (2014).

63. Isken, 0. et al. Upf1 phosphorylation triggers translational repression during nonsense-mediated mRNA decay. Cell 133, 314-327 (2008).

64. Gatfield, D. \& Izaurralde, E. Nonsense-mediated messenger RNA decay is initiated by endonucleolytic cleavage in Drosophila. Nature 429, 575-578 (2004).

65. Glavan, F., Behm-Ansmant, I., Izaurralde, E. \& Conti, E. Structures of the PIN domains of SMG6 and SMG5 reveal a nuclease within the mRNA surveillance complex. EMBO J. 25, 5117-5125 (2006). 
66. Huntzinger, E., Kashima, I., Fauser, M., Sauliere, J. \& Izaurralde, E. SMG6 is the catalytic endonuclease that cleaves mRNAs containing nonsense codons in metazoan. RNA 14, 2609-2617 (2008).

67. Eberle, A. B., Lykke-Andersen, S., Muhlemann, O. \& Jensen, T. H. SMG6 promotes endonucleolytic cleavage of nonsense mRNA in human cells. Nat. Struct. Mol. Biol. 16, 49-55 (2009).

68. Schmidt, S. A. et al. Identification of SMG6 cleavage sites and a preferred RNA cleavage motif by global analysis of endogenous NMD targets in human cells. Nucleic Acids Res. (2014).

69. Lykke-Andersen, S. et al. Human nonsense-mediated RNA decay initiates widely by endonucleolysis and targets snoRNA host genes. Genes Dev. 28, 24982517 (2014).

References 68 and 69 used transcriptome-wide approaches that identify NMD-responsive mRNAs and NMD-specific degradation intermediates to improve the chance of finding direct NMD targets.

70. Unterholzner, L. \& Izaurralde, E. SMG7 acts as a molecular link between mRNA surveillance and mRNA decay. Mol. Cell 16, 587-596 (2004).

71. Yamashita, A. et al. Concerted action of poly(A) nucleases and decapping enzyme in mammalian mRNA turnover. Nat. Struct. Mol. Biol. 12, 1054-1063 (2005).

72. Loh, B., Jonas, S. \& Izaurralde, E. The SMG5-SMG7 heterodimer directly recruits the CCR4-NOT deadenylase complex to mRNAs containing nonsense codons via interaction with POP2. Genes Dev. 27, 2125-2138 (2013).

73. Cho, H., Kim, K. M. \& Kim, Y. K. Human proline-rich nuclear receptor coregulatory protein 2 mediates an interaction between mRNA surveillance machinery and decapping complex. Mol. Cell 33, 75-86 (2009).

74. Lai, T. et al. Structural basis of the PNRC2-mediated link between mrna surveillance and decapping. Structure 20, 2025-2037 (2012).

75. Cho, H. et al. SMG5-PNRC2 is functionally dominant compared with SMG5SMG7 in mammalian nonsense-mediated mRNA decay. Nucleic Acids Res. 41, 1319-1328 (2013).

76. Anders, K. R., Grimson, A. \& Anderson, P. SMG-5, required for C.elegans nonsense-mediated mRNA decay, associates with SMG-2 and protein phosphatase 2A. EMBO J. 22, 641-650 (2003).

77. Durand, S. et al. Inhibition of nonsense-mediated mRNA decay (NMD) by a new chemical molecule reveals the dynamic of NMD factors in P-bodies. J. Cell Biol. 178, 1145-1160 (2007).

78. Mendell, J. T., Sharifi, N. A., Meyers, J. L., Martinez-Murillo, F. \& Dietz, H. C. Nonsense surveillance regulates expression of diverse classes of mammalian transcripts and mutes genomic noise. Nat. Genet. 36, 1073-1078 (2004).

79. Wittmann, J., Hol, E. M. \& Jack, H. M. hUPF2 silencing identifies physiologic substrates of mammalian nonsense-mediated mRNA decay. Mol. Cell. Biol. 26, 1272-1287 (2006). 
80. Ni, J. Z. et al. Ultraconserved elements are associated with homeostatic control of splicing regulators by alternative splicing and nonsense-mediated decay. Genes Dev. 21, 708-718 (2007).

81. Saltzman, A. L. et al. Regulation of multiple core spliceosomal proteins by alternative splicing-coupled nonsense-mediated mRNA decay. Mol. Cell. Biol. 28, 4320-4330 (2008).

82. Weischenfeldt, J. et al. NMD is essential for hematopoietic stem and progenitor cells and for eliminating by-products of programmed DNA rearrangements. Genes Dev. 22, 1381-1396 (2008).

83. Thoren, L. A. et al. UPF2 is a critical regulator of liver development, function and regeneration. PLoS One 5, e11650 (2010).

84. McIlwain, D. R. et al. Smg1 is required for embryogenesis and regulates diverse genes via alternative splicing coupled to nonsense-mediated mRNA decay. Proc. Natl. Acad. Sci. U. S. A. 107, 12186-12191 (2010).

85. Wang, D. et al. Inhibition of nonsense-mediated RNA decay by the tumor microenvironment promotes tumorigenesis. Mol. Cell. Biol. 31, 3670-3680 (2011).

86. Weischenfeldt, J. et al. Mammalian tissues defective in nonsense-mediated mRNA decay display highly aberrant splicing patterns. Genome Biol. 13, R352012-13-5-r35 (2012).

References 82,83 and 86 demonstrate the roles of NMD in various physiological processes.

87. Tani, H. et al. Identification of hundreds of novel UPF1 target transcripts by direct determination of whole transcriptome stability. RNA Biol. 9, 1370-1379 (2012).

References 85 and 87 measured mRNA half-lives in control and NMDinhibited conditions to identify direct targets of NMD.

88. Nguyen, L. S. et al. Transcriptome profiling of UPF3B/NMD-deficient lymphoblastoid cells from patients with various forms of intellectual disability. Mol. Psychiatry 17, 1103-1115 (2012).

89. Nguyen, L. S. et al. Contribution of copy number variants involving nonsensemediated mRNA decay pathway genes to neuro-developmental disorders. Hum. Mol. Genet. 22, 1816-1825 (2013).

90. Wong, J. J. et al. Orchestrated intron retention regulates normal granulocyte differentiation. Cell 154, 583-595 (2013).

91. Gardner, L. B. Hypoxic inhibition of nonsense-mediated RNA decay regulates gene expression and the integrated stress response. Mol. Cell. Biol. 28, 37293741 (2008).

92. Martin, L. \& Gardner, L. B. Stress-induced inhibition of nonsense-mediated RNA decay regulates intracellular cystine transport and intracellular glutathione through regulation of the cystine/glutamate exchanger SLC7A11. Oncogene http://dx.doi.org/10.1038/onc.2014.352 (2014).

93. Oren, Y. S. et al. The unfolded protein response affects readthrough of premature termination codons. EMBO Mol. Med. 6, 685-701 (2014). 
94. Karam, R. et al. The unfolded protein response is shaped by the NMD pathway. EMBO Rep. 16, 599-609 (2015).

This reference provides evidence that NMD shapes the cellular threshold of endoplasmic reticulum stress by restricting the levels of mRNAs encoding proteins involved in the unfolded protein response (UPR).

95. Lareau, L. F., Inada, M., Green, R. E., Wengrod, J. C. \& Brenner, S. E. Unproductive splicing of SR genes associated with highly conserved and ultraconserved DNA elements. Nature 446, 926-929 (2007).

References 80, 81, 86 and 95 describe the widespread use of AS-NMD in transcripts of splicing factors. Reference 86 shows that some splicing factors are misregulated in UPF2-deficient mouse cells, which leads to a general increase in alternative splicing.

96. Mourtada-Maarabouni, M. \& Williams, G. T. Growth arrest on inhibition of nonsense-mediated decay is mediated by noncoding RNA GAS5. Biomed. Res. Int. 2013, 358015 (2013).

97. Tani, H., Torimura, M. \& Akimitsu, N. The RNA degradation pathway regulates the function of GAS5 a non-coding RNA in mammalian cells. PLoS One 8, e55684 (2013).

98. Askarian-Amiri, M. E. et al. SNORD-host RNA Zfas1 is a regulator of mammary development and a potential marker for breast cancer. RNA 17, 878-891 (2011).

99. Kadlec, J., Guilligay, D., Ravelli, R. B. \& Cusack, S. Crystal structure of the UPF2-interacting domain of nonsense-mediated mRNA decay factor UPF1. RNA 12, 1817-1824 (2006).

100. Takahashi, S. et al. Upf1 potentially serves as a RING-related E3 ubiquitin ligase via its association with Upf3 in yeast. RNA 14, 1950-1958 (2008).

101. Kuroha, K., Tatematsu, T. \& Inada, T. Upf1 stimulates degradation of the product derived from aberrant messenger RNA containing a specific nonsense mutation by the proteasome. EMBO Rep. 10, 1265-1271 (2009).

102. Kuroha, K., Ando, K., Nakagawa, R. \& Inada, T. The Upf factor complex interacts with aberrant products derived from mRNAs containing a premature termination codon and facilitates their proteasomal degradation. J. Biol. Chem. 288, 28630-28640 (2013).

103. Maquat, L. E., Hwang, J., Sato, H. \& Tang, Y. CBP80-promoted mRNP rearrangements during the pioneer round of translation, nonsense-mediated mRNA decay, and thereafter. Cold Spring Harb. Symp. Quant. Biol. 75, 127-134 (2010).

104. Durand, S. \& Lykke-Andersen, J. Nonsense-mediated mRNA decay occurs during eIF4F-dependent translation in human cells. Nat. Struct. Mol. Biol. 20, 702-709 (2013).

105. Rufener, S. C. \& Muhlemann, O. eIF4E-bound mRNPs are substrates for nonsense-mediated mRNA decay in mammalian cells. Nat. Struct. Mol. Biol. 20, 710-717 (2013). 
106. Singh, G., Jakob, S., Kleedehn, M. G. \& Lykke-Andersen, J. Communication with the exon-junction complex and activation of nonsense-mediated decay by human Upf proteins occur in the cytoplasm. Mol. Cell 27, 780-792 (2007).

107. Miller, J. N. \& Pearce, D. A. Nonsense-mediated decay in genetic disease: friend or foe? Mutat. Res. Rev. Mutat. Res. 762, 52-64 (2014).

108. Holbrook, J. A., Neu-Yilik, G., Hentze, M. W. \& Kulozik, A. E. Nonsensemediated decay approaches the clinic. Nat. Genet. 36, 801-808 (2004).

109. Khajavi, M., Inoue, K. \& Lupski, J. R. Nonsense-mediated mRNA decay modulates clinical outcome of genetic disease. Eur. J. Hum. Genet. 14, 1074-1081 (2006).

110. Miller, J. in Molecular Genetic Pathology (eds Cheng, L., Zhang, D. Y. \& Eble, J. N.) 825-856 (Springer New York, 2012).

111. Li, S. \& Wilkinson, M. F. Nonsense surveillance in lymphocytes? Immunity 8 , 135-141 (1998).

112. Ge, Y. \& Porse, B. T. The functional consequences of intron retention: Alternative splicing coupled to NMD as a regulator of gene expression. Bioessays (2013).

113. Lareau, L. F., Brooks, A. N., Soergel, D. A., Meng, Q. \& Brenner, S. E. The coupling of alternative splicing and nonsense-mediated mRNA decay. Adv. Exp. Med. Biol. 623, 190-211 (2007).

114. Malabat, C., Feuerbach, F., Ma, L., Saveanu, C. \& Jacquier, A. Quality control of transcription start site selection by nonsense-mediated-mRNA decay. Elife 4, 10.7554/eLife.06722 (2015).

115. Colak, D., Ji, S. J., Porse, B. T. \& Jaffrey, S. R. Regulation of axon guidance by compartmentalized nonsense-mediated mRNA decay. Cell 153, 1252-1265 (2013).

This reference demonstrates that proper spatial expression of the ROBO3.2 receptor is controlled by localized translation and NMD-targeting of the Robo3.2 mRNA in certain axonal growth cones.

116. Sureau, A., Gattoni, R., Dooghe, Y., Stevenin, J. \& Soret, J. SC35 autoregulates its expression by promoting splicing events that destabilize its mRNAs. EMBO J. 20, 1785-1796 (2001).

117. Wollerton, M. C., Gooding, C., Wagner, E. J., Garcia-Blanco, M. A. \& Smith, C. W. Autoregulation of polypyrimidine tract binding protein by alternative splicing leading to nonsense-mediated decay. Mol. Cell 13, 91-100 (2004).

118. Braunschweig, U. et al. Widespread intron retention in mammals functionally tunes transcriptomes. Genome Res. 24, 1774-1786 (2014).

119. Somers, J., Poyry, T. \& Willis, A. E. A perspective on mammalian upstream open reading frame function. Int. J. Biochem. Cell Biol. 45, 1690-1700 (2013).

120. Ramani, A. K. et al. High resolution transcriptome maps for wild-type and nonsense-mediated decay-defective Caenorhabditis elegans. Genome Biol. 10, R101-2009-10-9-r101. Epub 2009 Sep 24 (2009). 
121. Vattem, K. M. \& Wek, R. C. Reinitiation involving upstream ORFs regulates ATF4 mRNA translation in mammalian cells. Proc. Natl. Acad. Sci. U. S. A. 101, 11269-11274 (2004).

122. Dinman, J. D. Mechanisms and implications of programmed translational frameshifting. Wiley Interdiscip. Rev. RNA 3, 661-673 (2012).

123. Belew, A. T. et al. Ribosomal frameshifting in the CCR5 mRNA is regulated by miRNAs and the NMD pathway. Nature 512, 265-269 (2014).

124. Shetty, S. P. \& Copeland, P. R. Selenocysteine incorporation: A trump card in the game of mRNA decay. Biochimie 114, 97-101 (2015).

125. Sunde, R. A. \& Raines, A. M. Selenium regulation of the selenoprotein and nonselenoprotein transcriptomes in rodents. Adv. Nutr. 2, 138-150 (2011).

126. Seyedali, A. \& Berry, M. J. Nonsense-mediated decay factors are involved in the regulation of selenoprotein mRNA levels during selenium deficiency. RNA 20, 1248-1256 (2014).

127. Usuki, F., Yamashita, A. \& Fujimura, M. Post-transcriptional defects of antioxidant selenoenzymes cause oxidative stress under methylmercury exposure. J. Biol. Chem. 286, 6641-6649 (2011).

128. Medghalchi, S. M. et al. Rent1, a trans-effector of nonsense-mediated mRNA decay, is essential for mammalian embryonic viability. Hum. Mol. Genet. 10, 99105 (2001).

129. Li, T. et al. Smg6/Est1 licenses embryonic stem cell differentiation via nonsense-mediated mRNA decay. EMBO J. (2015).

This reference shows that viable SMG6-deficient embryonic stem cells fail to differentiate and that NMD, and not telomere maintenance, is the critical activity underlying this defect.

130. Imamachi, N., Tani, H. \& Akimitsu, N. Up-frameshift protein 1 (UPF1): multitalented entertainer in RNA decay. Drug Discov. Ther. 6, 55-61 (2012).

131. Varsally, W. \& Brogna, S. UPF1 involvement in nuclear functions. Biochem. Soc. Trans. 40, 778-783 (2012).

132. Kaygun, H. \& Marzluff, W. F. Regulated degradation of replicationdependent histone mRNAs requires both ATR and Upf1. Nat. Struct. Mol. Biol. 12, 794-800 (2005).

133. Gong, C., Kim, Y. K., Woeller, C. F., Tang, Y. \& Maquat, L. E. SMD and NMD are competitive pathways that contribute to myogenesis: effects on PAX3 and myogenin mRNAs. Genes Dev. 23, 54-66 (2009).

134. Jolly, L. A., Homan, C. C., Jacob, R., Barry, S. \& Gecz, J. The UPF3B gene, implicated in intellectual disability, autism, ADHD and childhood onset schizophrenia regulates neural progenitor cell behaviour and neuronal outgrowth. Hum. Mol. Genet. 22, 4673-4687 (2013).

135. Lou, C. H. et al. Posttranscriptional control of the stem cell and neurogenic programs by the nonsense-mediated RNA decay pathway. Cell. Rep. 6, 748-764 (2014). 
136. Bruno, I. G. et al. Identification of a microRNA that activates gene expression by repressing nonsense-mediated RNA decay. Mol. Cell 42, 500-510 (2011).

References 135 and 136 demonstrate that specific microRNAs target NMD factors to downregulate the activity of NMD, and that this is necessary for the differentiation of neuronal progenitor cells into neurons.

137. Preitner, N., Quan, J. \& Flanagan, J. G. This message will self-destruct: NMD regulates axon guidance. Cell 153, 1185-1187 (2013).

138. Tarpey, P. S. et al. Mutations in UPF3B, a member of the nonsense-mediated mRNA decay complex, cause syndromic and nonsyndromic mental retardation. Nat. Genet. 39, 1127-1133 (2007).

139. Yamasaki, S. \& Anderson, P. Reprogramming mRNA translation during stress. Curr. Opin. Cell Biol. 20, 222-226 (2008).

140. Nickless, A. et al. Intracellular calcium regulates nonsense-mediated mRNA decay. Nat. Med. 20, 961-966 (2014).

141. Brown, J. A. et al. A novel role for hSMG-1 in stress granule formation. Mol. Cell. Biol. 31, 4417-4429 (2011).

142. Park, E. \& Maquat, L. E. Staufen-mediated mRNA decay. Wiley Interdiscip. Rev. RNA 4, 423-435 (2013).

143. Chakrabarti, S. et al. Molecular mechanisms for the RNA-dependent ATPase activity of Upf1 and its regulation by Upf2. Mol. Cell 41, 693-703 (2011).

144. Clerici, M. et al. Unusual bipartite mode of interaction between the nonsense-mediated decay factors, UPF1 and UPF2. EMBO J. 28, 2293-2306 (2009).

145. Fiorini, F., Boudvillain, M. \& Le Hir, H. Tight intramolecular regulation of the human Upf1 helicase by its $\mathrm{N}$ - and C-terminal domains. Nucleic Acids Res. 41, 2404-2415 (2013).

\section{Acknowledgements}

We apologize to those researchers whose work did not get cited due to size limitations. We thank Christian Kroun Damgaard, Jens Lykke-Andersen and four insightful reviewers for critical assessments of the manuscript. Research in the authors' laboratory is funded by The European Research Council, and The Danish National Research- (grant DNRF58), The Lundbeck- and The Novo Nordisk Foundations. 


\section{Competing interests statement}

The authors have no competing interests as defined by Nature Publishing Group, or other interests that might be perceived to influence the interpretation of the article.

\section{Display items}

\section{Box 1 | Identification of direct targets of NMD}

Transcriptome-wide identification of direct NMD targets is challenging. A typical experimental setup involves depletion of a NMD factor and comparing the reshaped transcriptome to that of an untreated cell population by either microarray or next generation sequencing analyses ${ }^{18,49,68,69,78-90}$. mRNAs detected at increased levels when NMD is compromised are putative substrates of NMD. However, stabilized mRNAs may encode proteins involved in other aspects of RNA metabolism, and, consequently, levels of mRNAs that are not direct NMD targets may change. Additionally, several NMD factors have NMD-independent roles as exemplified by the role of UPF1 in telomere maintenance, histone mRNA degradation and Staufen1-mediated decay ${ }^{130,131,142}$. Manual inspection for NMDactivating features is often used to support the identification of direct targets, but since the full scope of such features is not yet complete alternative or additional experimental strategies should be employed. In one approach, mRNA half-lives were compared between UPF1 depleted and control cells. This enabled the detection of hundreds of mRNAs with increased half-lives ${ }^{85,87}$. In another approach mRNAs whose levels increased after NMD inhibition were sorted for those also displaying evidence for SMG6-catalyzed endonucleolytic cleavage. Again, hundreds of NMD substrates were found ${ }^{68,69}$. The mentioned approaches still rely on prolonged depletion of proteins, which may not always be practical especially when studying dynamic processes such as cellular differentiation. In such cases, an experimental setup minimizing the risk of interfering with the inspected process is preferable. This can for example be achieved by capturing NMD targeted mRNAs by immunoprecipitation of a central NMD factor. Whereas hypophosphorylated UPF1 interacts with both mRNA substrates and non- 
substrates of NMD ${ }^{11,23-27}$, carboxy-terminal phosphorylation of UPF1 signifies a late step in NMD just before and when the mRNA is degraded (Fig. 1c). An antibody specifically recognizing carboxy-terminally phosphorylated UPF1 may therefore be a potential bait for extracting genuine NMD substrates from unperturbed cells ${ }^{24}$.

\section{Figure legends}

\section{Figure 1 | Normal and NMD-activating translation termination}

a | Efficient translation termination prevents NMD. Translation is efficiently terminated when the termination codon (TC) is in physical proximity to the $3^{\prime}$ poly(A) tail (AAAAAAAA) and/or the $5^{\prime}$ cap $\left(\mathrm{m}^{7} \mathrm{G}\right)$ of the mRNA. The release factor complex eRF1-eRF3 interacts with the termination codon and the ribosome. The cytoplasmic poly(A) binding protein (PABPC) and the 5' cap bound translation initiation factor eIF4G, associated to either the cap binding complex (CBC; not shown) or the cap binding translation initiation factors eIF4E-eIF4A1, interact to circularise the mRNA. This interaction allows efficient translation termination and prevents UPF1, which only binds transiently and nonspecifically to the mRNA, from interacting with eRF3 when the termination codon is proximal to the 3' and/or 5' ends of the mRNA. b | Recruitment of UPF1, UPF2 and UPF3 during inefficient translation termination. (1) UPF1 binds nonspecifically to the mRNA. (2) NMD is enabled when ribosome-associated eRF3 interacts with UPF1, thereby recruiting UPF2 and/or UPF3. (3) UPF2 and/or UPF3 recruitment is assisted by an exon junction complex (EJC) bound to the 3' untranslated region (UTR), but can also occur independently (4). Some mRNAs may escape NMD for one or more rounds of translation due to the inefficient recruitment of UPF1, UPF2 and/or UPF3 to the terminating ribosome. c | Assembly of a NMD-activating complex on a PTC-containing mRNA and initiation of decay. The termination codon is marked as premature (PTC) upon assembly of a protein complex including UPF1, UPF2, UPF3, SMG1, SMG8 (8), SMG9 (9), DHX34 and the EJC (top). At this point translation may terminate, ultimately leading to the dissociation of the individual ribosomal subunits, the 
release factors and the nascent protein. UPF2 binds to the amino-terminal domain of UPF1, releasing this domain from the central core of UPF1 ${ }^{143,144}$. SMG1 phosphorylates residues in the amino- and carboxy-terminal domains of UPF1 (asterisks), which enables the recruitment (bottom) of factors that initiate mRNA degradation by endonucleolytic cleavage (SMG6), deadenylation (CCR4-NOT via SMG5-SMG7) and/or decapping (decapping complex (DCPc) via PNRC2). The decapping complex may be recruited to UPF1 independently of UPF1 phosphorylation. Recruitment of factors to the phosphorylated carboxy-terminal of UPF1 was suggested to free this domain from the central core of UPF1145. Endonucleolytic cleavage, decapping and deadenylation are followed by complete mRNA degradation by general cellular 5'-3' and 3'-5' exonucleolytic activities (XRN1 and the RNA exosome, respectively; not shown).

\section{Figure 2 | Roles of NMD in heterozygous carriers of PTC-mutations}

NMD prevents several possible phenotypic outcomes (illustrated in the bottom right box) from a PTC-generating mutation. In heterozygous carriers (illustrated by a functional allele (left) and an allele with a PTC-introducing mutation (right)), total levels of functional protein are halved, which may either be sufficient or insufficient to retain normal function.

Figure 3 | Importance of NMD for maturation of sufficient populations of Tcells

Flow-diagram illustrating sequential programmed rearrangements of TCR- $\beta$ alleles during T-cell maturation. Each rearrangement has a probability of up to one-third to produce a functional allele (one-third of events are in-frame, but some of these may introduce in-frame termination codons by nucleotide insertions). If the first rearrangement produces a functional TCR- $\beta$ allele, T-cell maturation continues (left). However, if it produces a PTC-containing allele, a second rearrangement takes place. If this rearrangement is productive, NMD elimates the PTC-containing mRNAs, allowing T-cell maturation to continue (middle, left arrow). In NMD-inhibited T-cells, such cells fail to survive (middle, 
right dashed arrow). If both alleles contain PTCs after rearrangements, the Tcells die (right).

Figure 4 | Expression homeostasis of splicing activators and splicing repressors

Model illustrating proposed expression homeostasis of factors regulating alternative splicing. Splicing activators (orange ellipses) can stimulate alternative splicing events in their cognate pre-mRNAs (top), which produce NMD-targeted mRNA isoforms. Conversely, splicing repressors (purple ellipses) can repress alternative splicing events in their cognate pre-mRNAs (bottom), thereby producing NMD-targeted isoforms. Through this autoregulatory loop, the levels of the involved splicing regulatory factors are kept at equilibrium, which affects the alternative splicing patterns of all pre-mRNAs in the cell (middle).

Figure 5 | Translation of alternative ORFs can activate NMD

a | Translation of the ATF4 mRNA during normal and stress conditions. In the case of ATF4 mRNA, a short upstream open reading frame (uORF) is initially translated followed by downstream re-initiation of translation. In normal conditions (left) the translation of a second uORF is favored, leading to NMD and low levels of ATF4 protein. Under stress conditions (right) a translation shift to the third, ATF4-coding ORF is favored, which stabilizes ATF4 mRNA with a consequent rise in levels of the ATF4 transcription factor. This stimulates transcription of its target genes involved in the integrated stress response. $\mathbf{b}$ | Translation of CCR5 mRNA with (left) or without (right) miRNA-induced frameshifting. The RNA element - a pseudoknot - that induces programmed ribosomal frameshifting by one nucleotide in the $5^{\prime}$ direction is indicated. Productive translation without frameshifting leads to production of CCR5 protein. Non-productive translation due to frameshifting, which introduces a PTC, is stimulated by binding of miR-1224 to the pseudoknot, thereby stabilizing it and triggering NMD of the CCR5 mRNA. c | Model for the translation of selenoprotein coding mRNAs in the presence (left) or absence (right) of 
selenium. The orange box illustrates the selenocysteine insertion sequence (SECIS) element that directs incorporation of selenocysteine at the indicated UGA termination codon in the selenoprotein $W, 1$ (SEPW1) mRNA. When selenium levels are sufficient selenocysteine is incorporated and translation continues until the downstream UAA termination codon. This UAA codon is 18 nucleotides upstream of the last exon-exon junction, but because a ribosome covers $\sim 30$ nucleotides, a possible NMD-stimulating EJC is displaced during the first round of translation. When selenium levels are insufficient the UGA codon is recognized as a PTC and NMD is triggered.

\section{Figure 6 | Global and local modulation of NMD activity}

a | Model for the regulation of NMD activity during the differentiation of neural progenitor cells into mature neurons. High NMD activity in progenitor cells ensures low levels of mRNAs encoding pro-neural factors and proliferation inhibitors, which sustains self-renewal of the stem cells. Upon induction of neuronal differentiation, levels of miRNAs that target NMD factors rise leading to downregulation of NMD and stabilization of the abovementioned mRNAs. This results in reduced proliferation and differentiation. b | Local effect of NMD in neuronal growth cones. (left) Schematic drawing of a commissural neuron. NMD factors are enriched in the axonal growth cone, resulting in local high NMD activity. (right) Before the growth cone passes the midline floorplate of the spinal cord, the translation of a PTC-containing Robo3.2 mRNA is repressed by an unknown mechanism. Following passage of the midline, translation is activated by floorplate-derived signals for one, or a few, round(s) before the mRNA is degraded by NMD. c | Model for NMD regulation during the unfolded protein response (UPR). Under normal conditions (left) levels of mRNAs encoding IRE1 $\alpha$ and other UPR-related proteins are restricted by NMD. NMD-mediated control of IRE1 $\alpha$ expression prevents initiation of the UPR under mild endoplasmic reticulum (ER) stress conditions. Following elevation in ER stress (right), global translation is downregulated whereas Ire1 $\alpha$ and other UPR-related mRNAs remain translated, but less susceptible to NMD, to mediate the UPR. NMD is 
important for efficient termination of the UPR and the reinstalling of normal conditions.

\section{Glossary}

Exon junction complex (EJC): A complex deposited upstream of most exon-exon junctions following splicing. The EJC mediates further mRNA processing and export.

Deadenylase: A 3'-5' exoribonuclease with specificity for poly(A)-moieties.

General decapping complex: A protein complex responsible for the hydrolysis of the $\mathrm{m}^{7}$-G-cap situated at mRNA $5^{\prime}$ ends.

$\beta$-thalassemia: Blood disorder caused by mutations in the $\beta$-globin $(H B B)$ gene on chromosome 11, which lead to reduced or absent production of hemoglobin beta chains.

Haploinsufficiency: A phenotype caused by the insufficient expression of a functional factor as a result of a mutation in one of the two encoding alleles.

Waardenburg syndrome type 4C: An auditory-pigmentary syndrome characterized by pigmentary abnormalities of the eye, deafness, and gastrointestinal disease.

Integrated stress response: A term covering various cellular stresses that act (partly) through the inactivation of the translation initiation factor subunit elF2 $\alpha$.

Programmed ribosomal frameshifting: A shift of the reading frame by one or two nucleotides (in the $5^{\prime}$ or $3^{\prime}$ direction) directed by a mRNA structural element.

Selenoproteins: Proteins containing one or more of the non-conventional amino acid selenocysteine.

Selenocysteine insertion sequence (SECIS): A 60 nt-long RNA sequence element situated downstream of a UGA termination codon in selenoprotein coding mRNAs.

Histone mRNA degradation: A UPF1-dependent degradation mechanism mediated by its binding of stem-loops at the $3^{\prime}$ end of replication-dependent histone mRNAs. 
Staufen1-mediated mRNA decay (SMD): Degradation of substrates with extended double-stranded 3' UTR structures that bind one, or both, of the UPF1-recruiting staufen paralogues STAU1 and STAU2.

Axonal growth cones: A dynamic extension at the tip of axons. Growth cones are able to rapidly change direction and branch in response to stimuli.

Commissural neurons: A tract of nerve fibers passing from one side to the other of the spinal cord or brain.

\section{Online summary}

- Nonsense-mediated mRNA decay (NMD) degrades mRNAs harboring translation termination codons in abnormal contexts.

- NMD targets mRNAs encoding truncated proteins with no or undesired functions, thereby preventing the accumulation of such transcripts and their encoded potentially aberrant proteins.

- NMD targets some mRNAs with intact open reading frames, thereby directly affecting gene expression programmes.

- NMD reduces the levels of mRNAs related to multiple normal cellular processes through its interplay with programmed DNA rearrangements, changes in premRNA processing or reprogramming of mRNA translation.

- NMD is essential for embryogenesis and other developmental processes.

- Up- and downregulation of NMD is essential for an efficient cellular response to stress and its subsequent alleviation, respectively.

\section{Author biographies}

Torben Heick Jensen has been on the Faculty of the Department of Molecular Biology and Genetics, Aarhus University, Denmark for 14 years. He carried out his doctoral work with Jørgen Kjems at Aarhus University, followed by postdoctoral studies with Michael Rosbash, HHMI, Brandeis, USA. His laboratory studies fundamental mechanisms and functions of RNA degradation and transcription in eukaryotic cells. 
Søren Lykke-Andersen is a senior post.doc. in Torben Heick Jensen's laboratory at the Department of Molecular Biology and Genetics, Aarhus University, Denmark. He carried out his doctoral work at Aarhus University with Jørgen Kjems. 
Figure 1

a

b

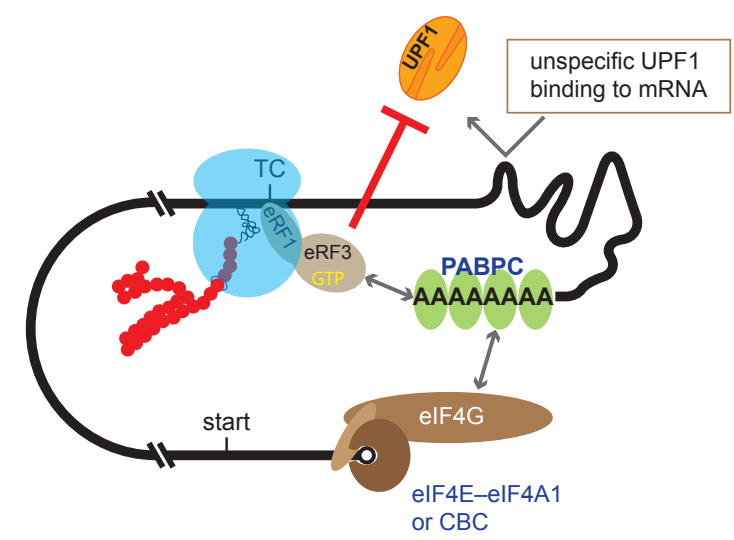

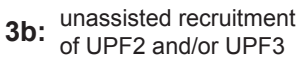

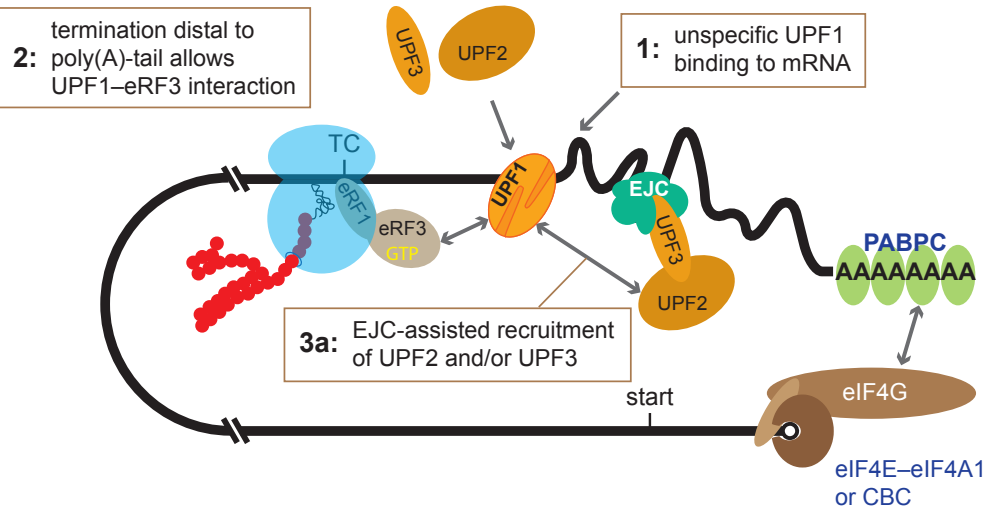

C
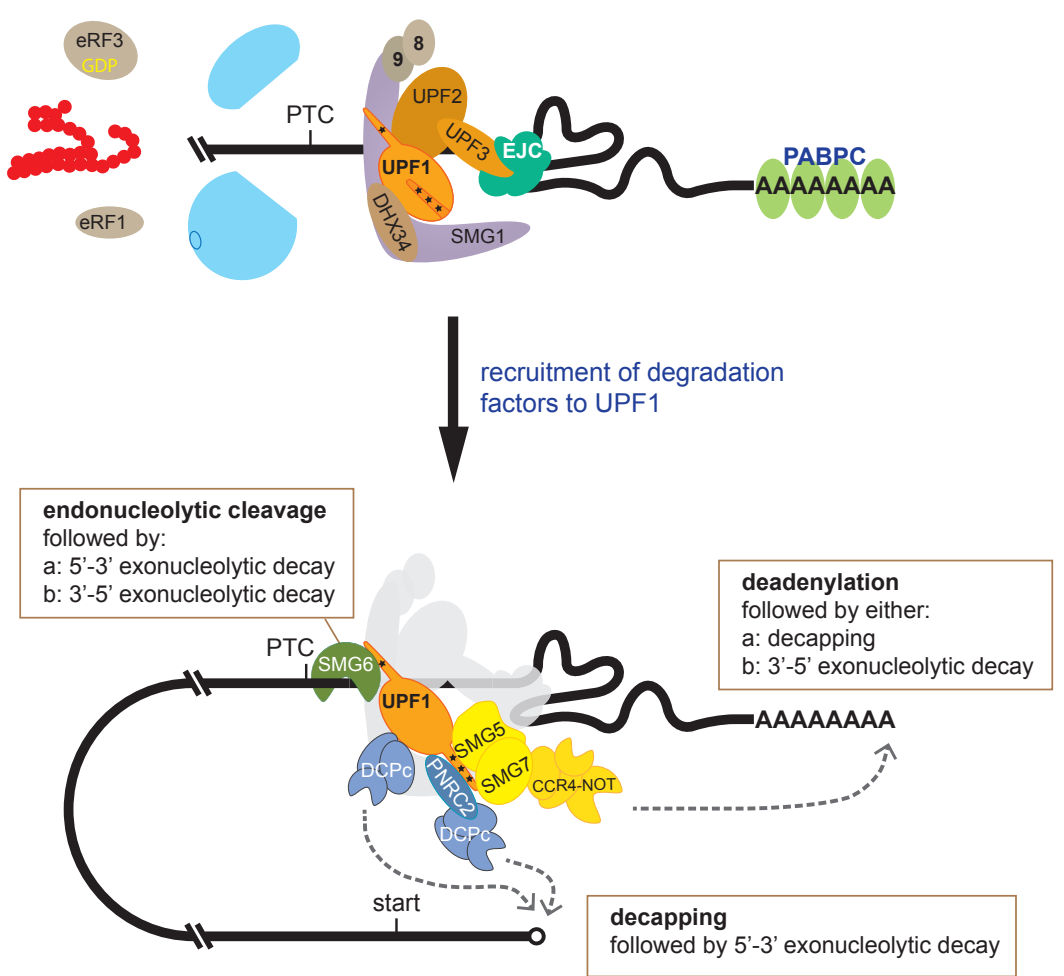


\section{Figure 2}

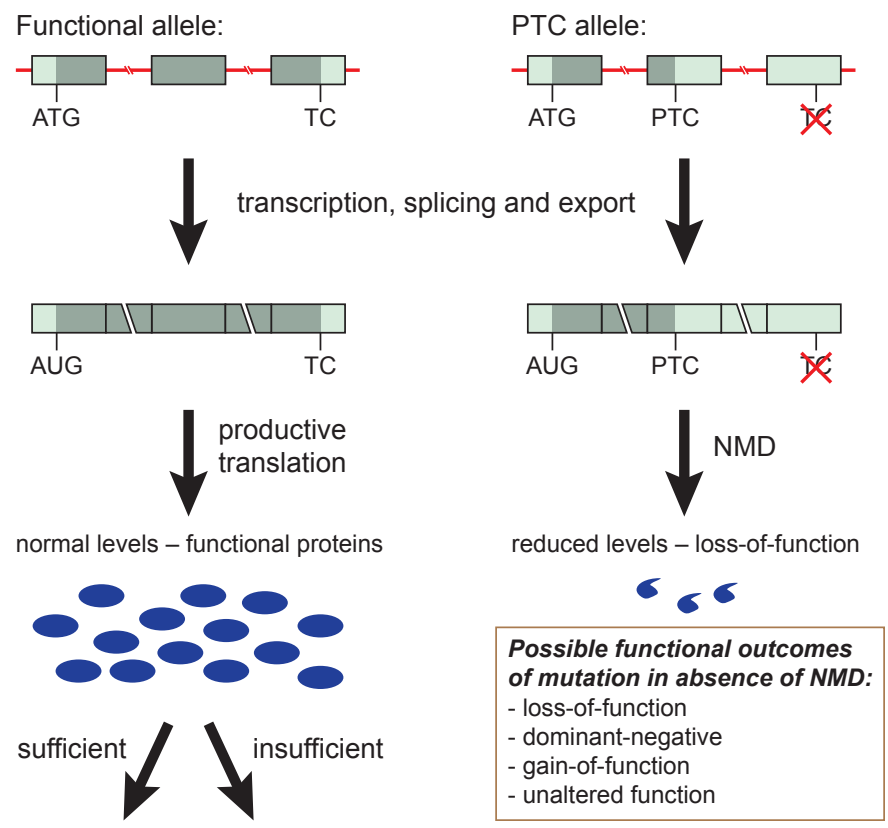

unaffected affected 
Figure 3

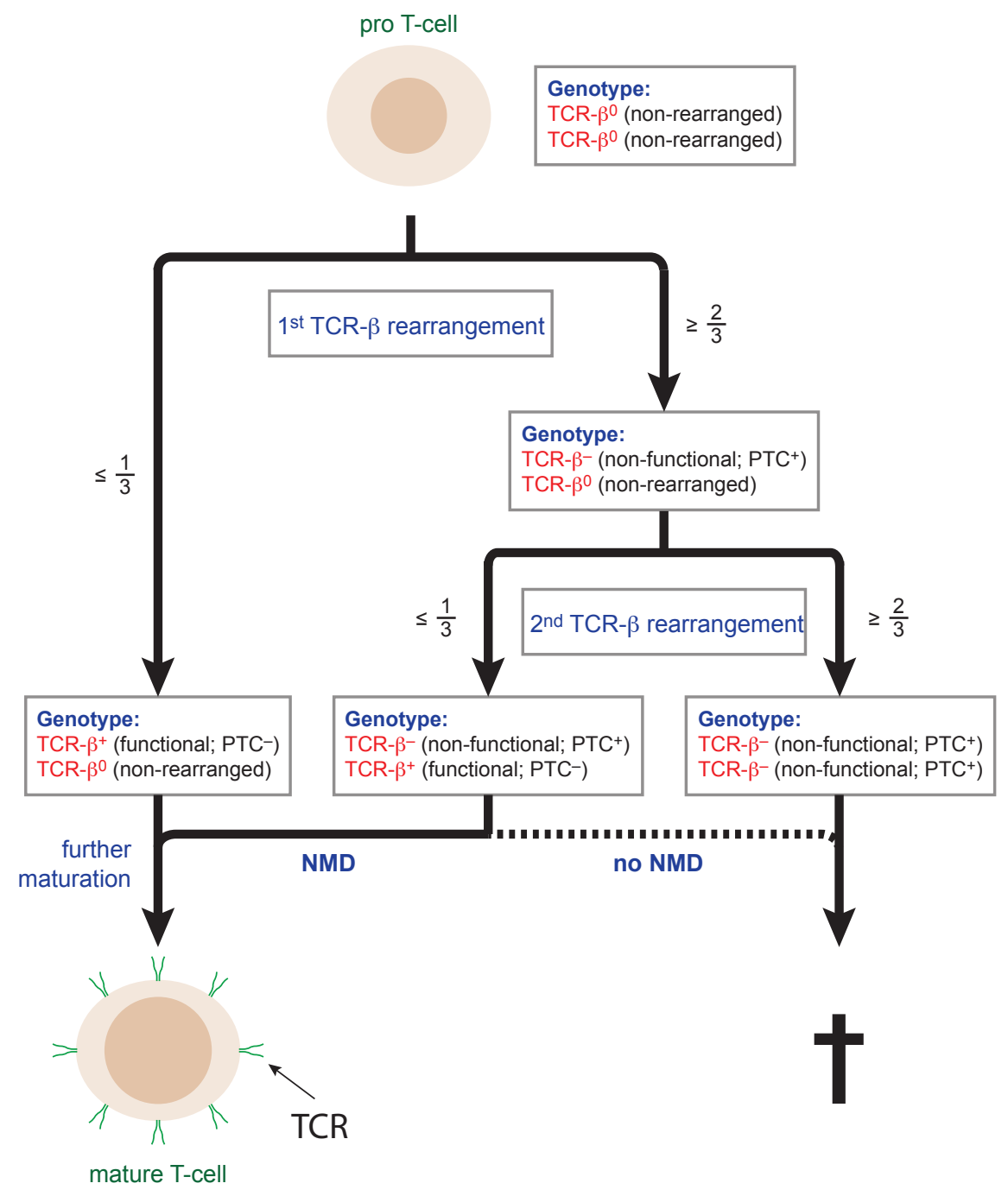


Figure 4

Splicing activator pre-mRNA

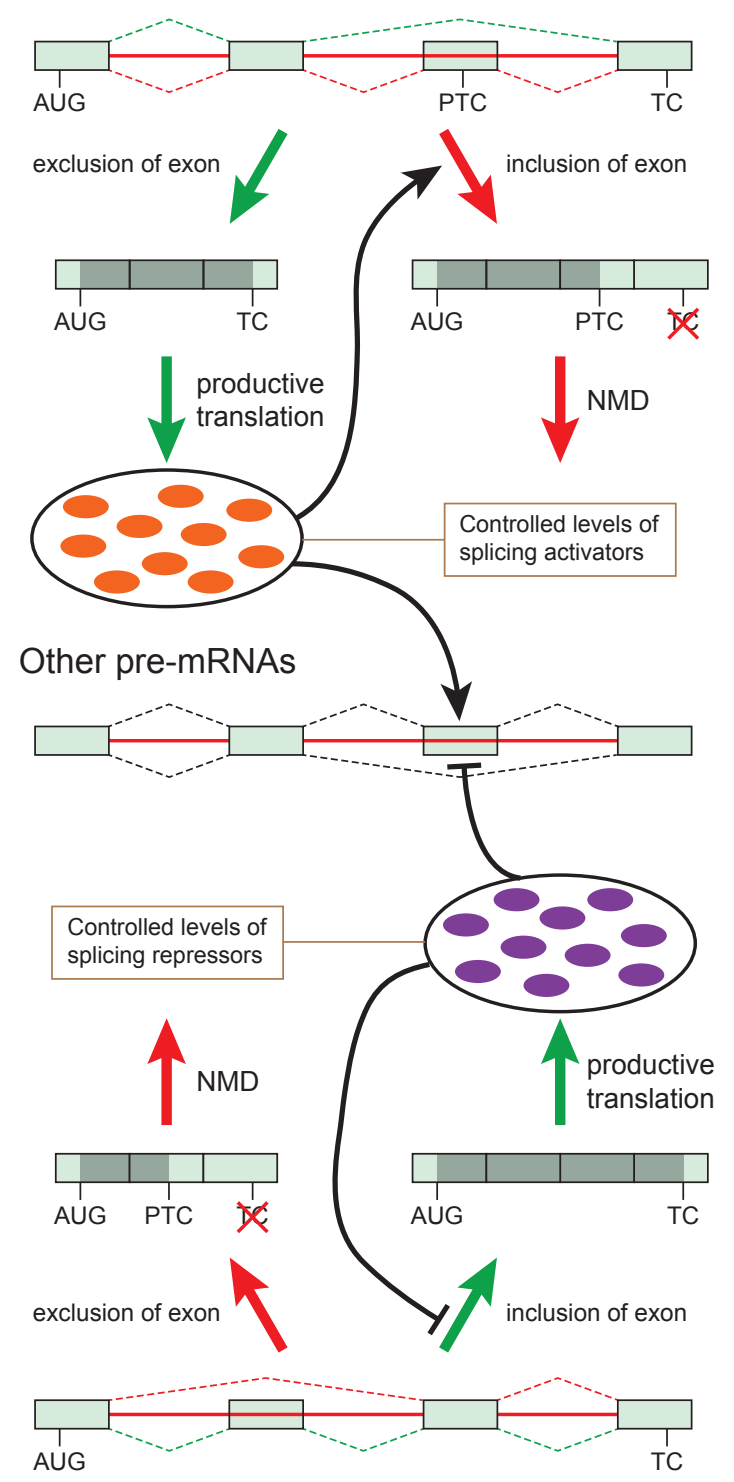

Splicing repressor pre-mRNA 


\section{Figure 5}

a

(1) Normal conditions

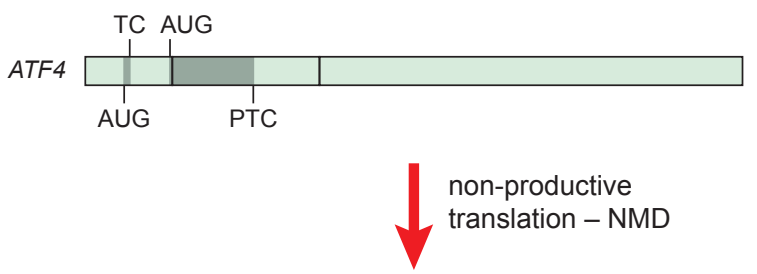

ATF4 mRNA degraded

no production of ATF4 protein

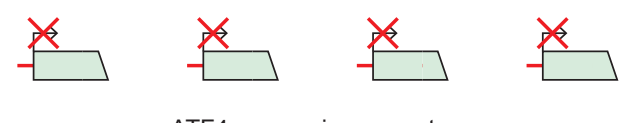

ATF4 responsive promoters

b

(1) No frameshifting

CCR5

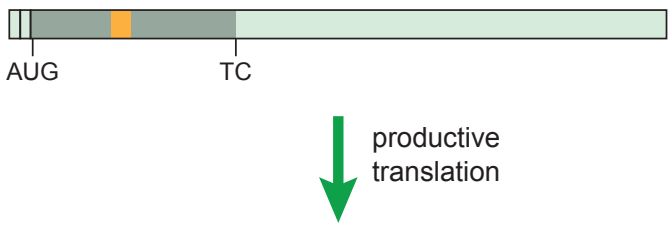

CCR5 mRNA stable

production of CCR5 protein

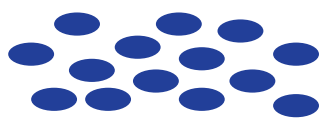

C

(1) Sufficient selenium

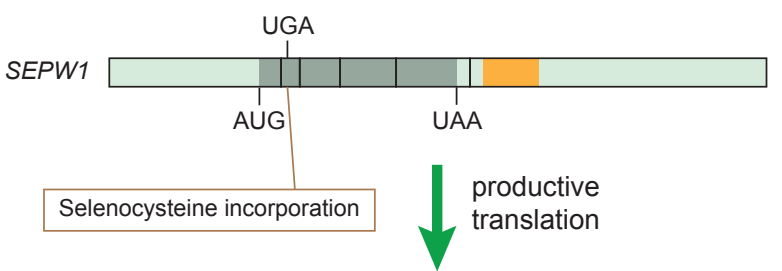

SEPW1 mRNA stable

production of SEPW1 protein

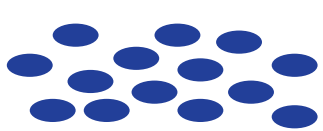

(2) Stress conditions

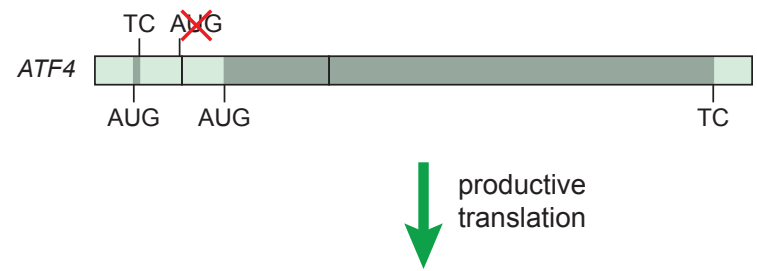

ATF4 mRNA stable

production of ATF4 protein

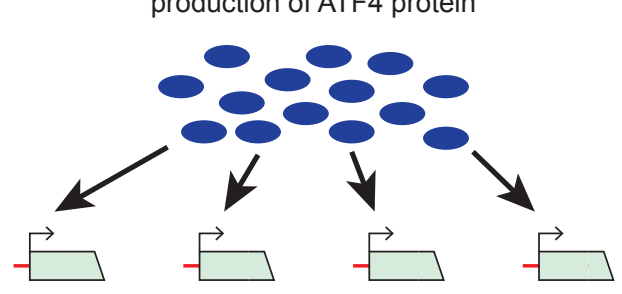

ATF4 responsive promoters

(2) miR-1224 induced frameshifting

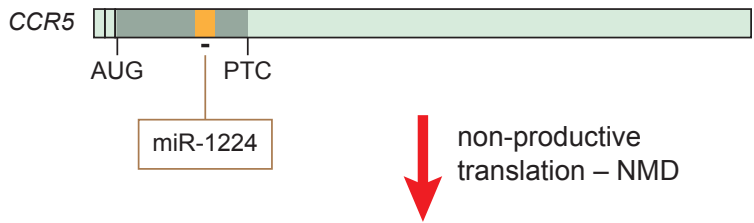

CCR5 mRNA degraded

no production of full-length CCR5 protein

(2) Insufficient selenium

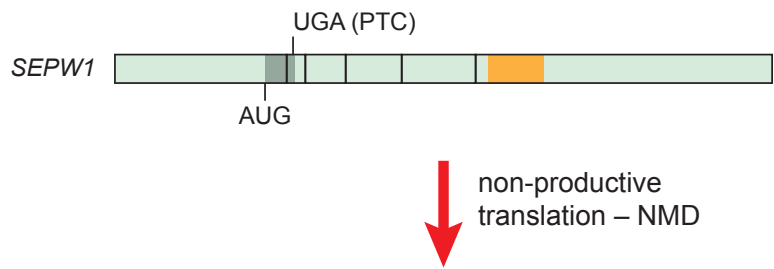

SEPW1 mRNA degraded

no production of SEPW1 protein 


\section{Figure 6}

a

neural progenitor cel

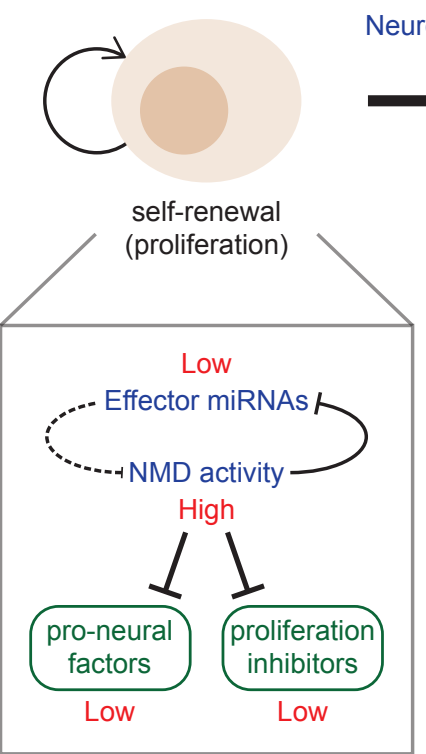

neuron

Neuronal differentiation signal

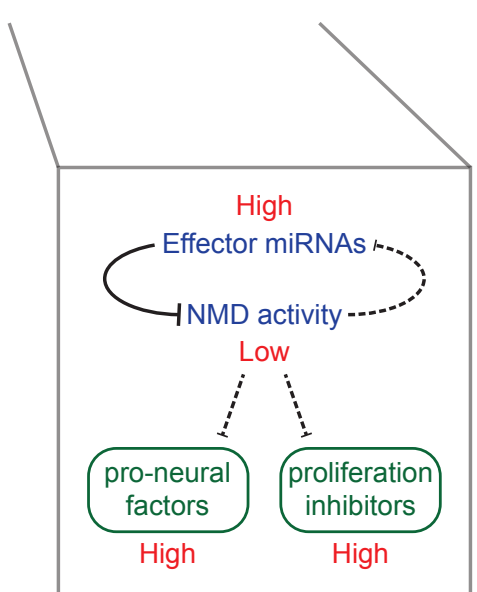

b

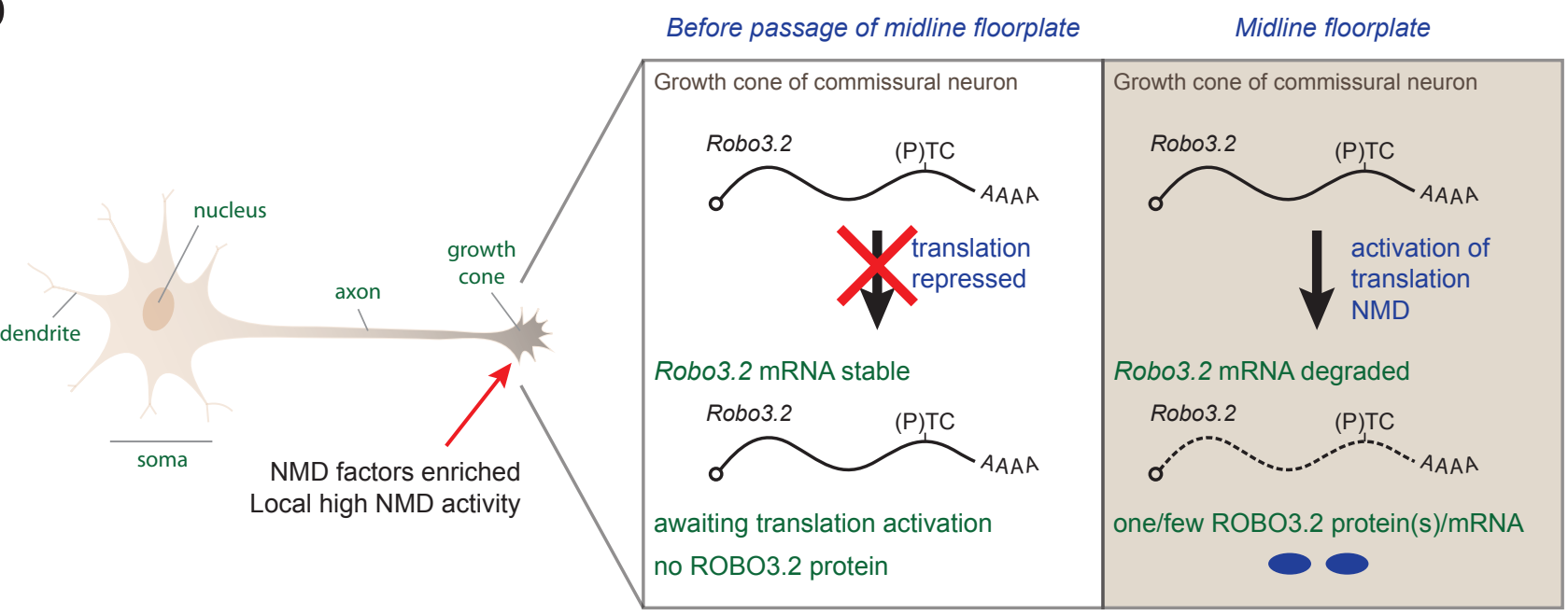

C

Normal conditions

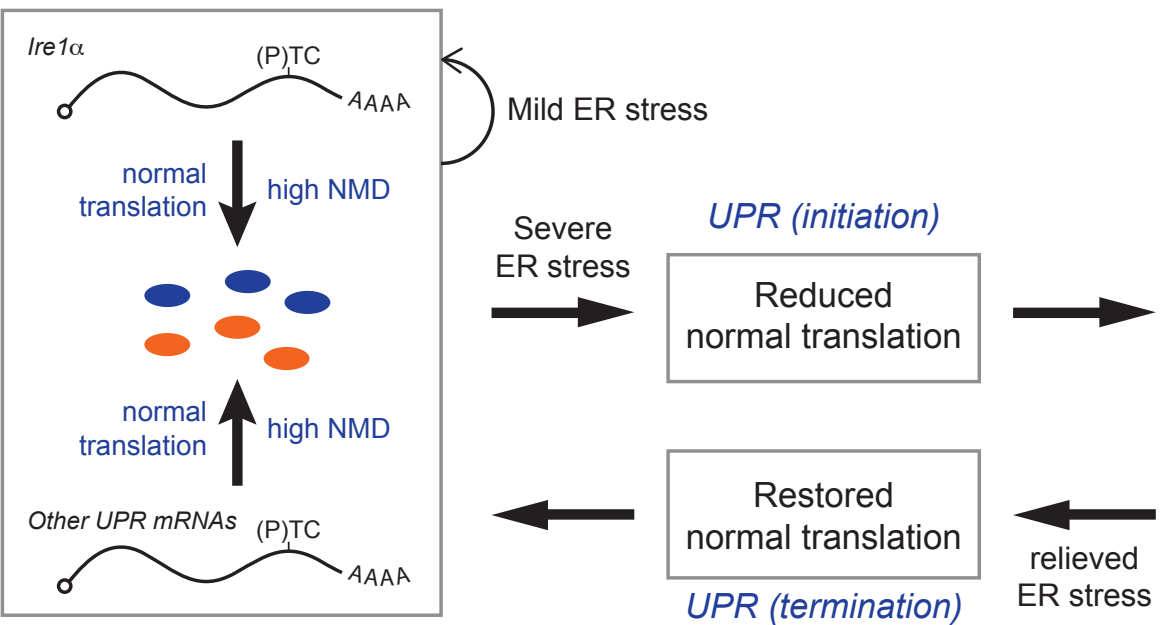

Unfolded protein response

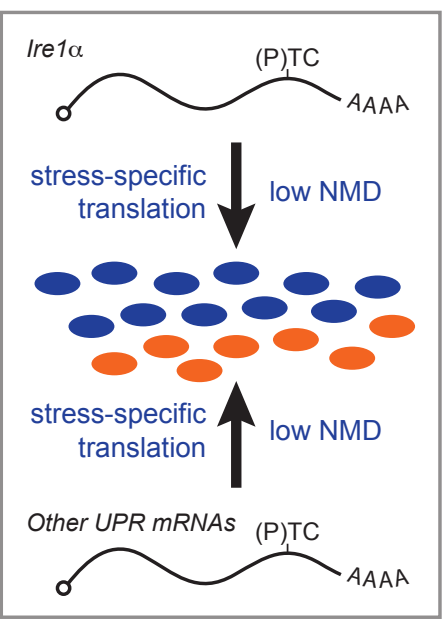




\section{Supplemental Figure 1}

a

(1)

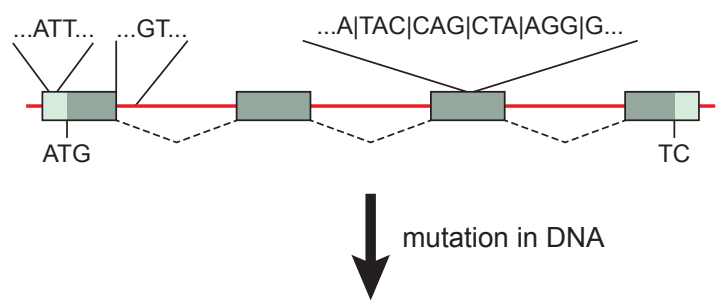

(2)

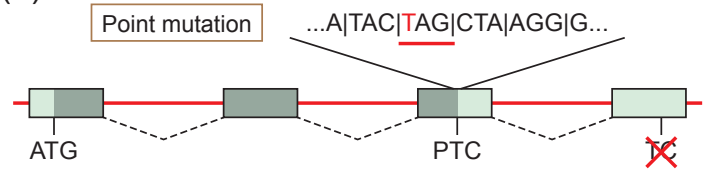

(3)

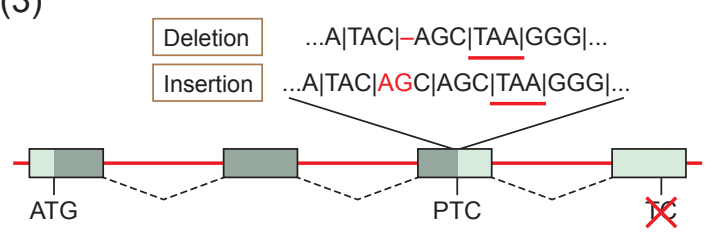

(4)

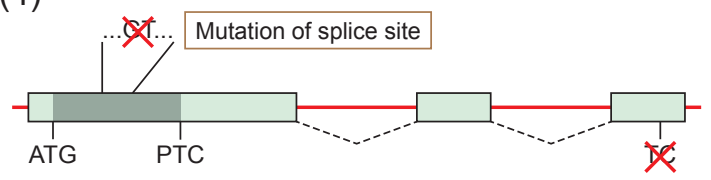

(5)

Mutation of translation start site

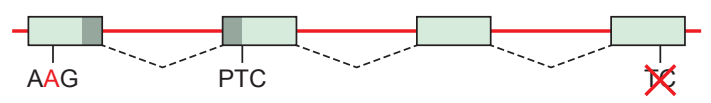

(6)

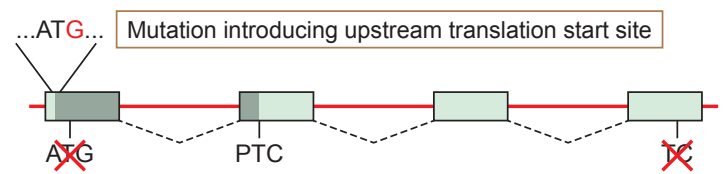

b

(1)

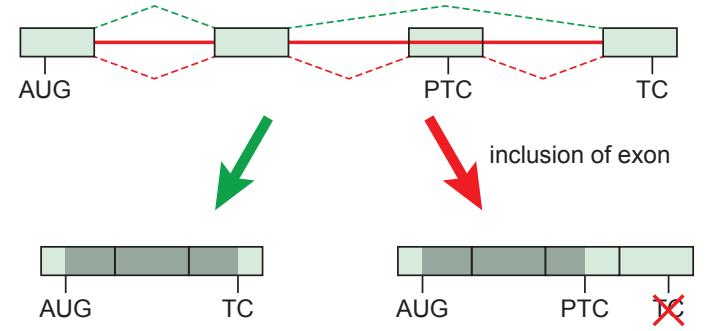

(2)

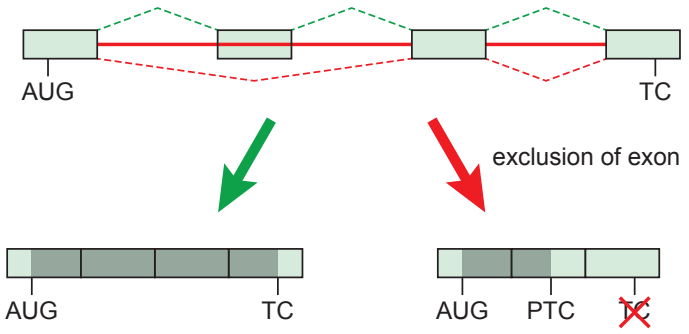

(3)
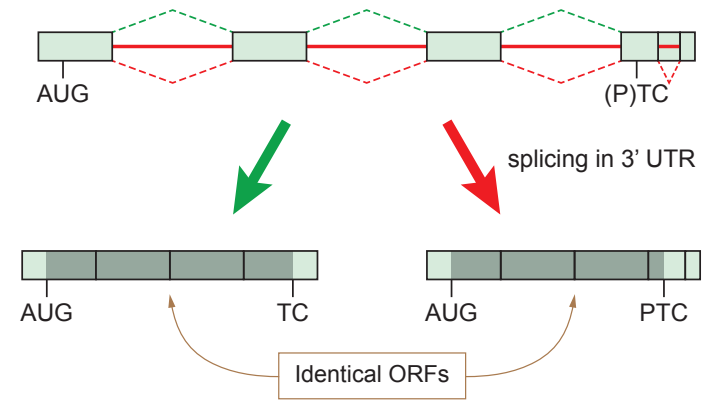

C

(1)

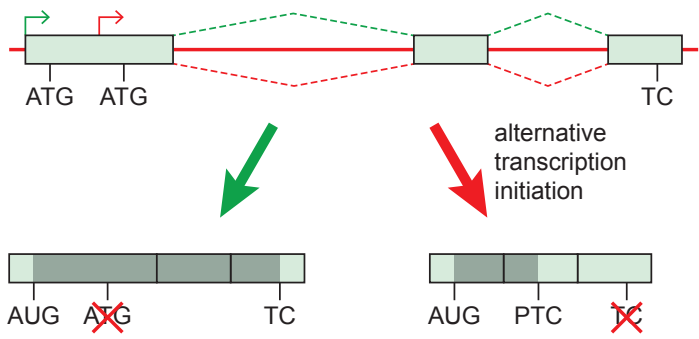

(2)

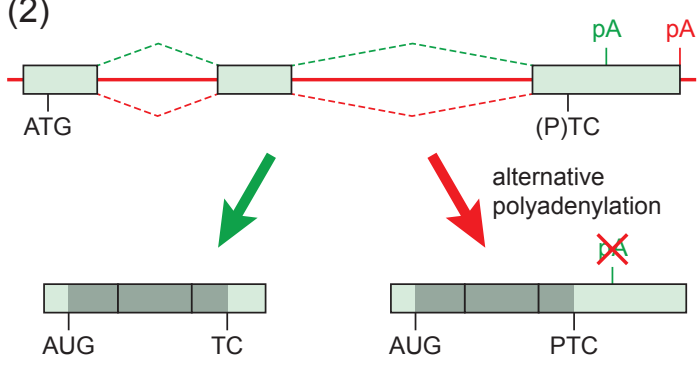




\section{Supplementary Figure S1}

\section{Supplemenrary Figure S1 | PTC-generating events}

a | Examples of mutations in protein-coding genes that can result in the production of PTC-containing mRNAs. (1) Schematic representation of an arbitrary gene with four exons (boxes) and three introns (red lines). The initiation (ATG) and termination codons (TC) are indicated. Untranslated and translated regions are depicted in light and dark green, respectively. pre-mRNA splicing events are indicated by dashed lines connecting the exons. The reading frames are indicated by | where applicable. (2) Point (nonsense) mutations introducing a PTC in the original open reading frame (ORF). Here illustrated by a C-to-T mutation (in red) changing a CAG glutamine codon to a TAG termination codon (red underline; the normal termination codon is no longer used). (3) Frameshift mutations (one- or two-base pair deletions or insertions) change the ORF and may introduce a PTC downstream of the mutation (deletion and insertion indicated in red by a dash and extra nucleotides, respectively). (4) Mutations leading to disruption or alteration of normal pre-mRNA splicing may introduce a PTC, for example by intron inclusion. (5) Mutations of translation initiation sites may cause translation to initiate at an out-of-frame, downstream ATG codon and introduce a PTC. (6) Mutations introducing an upstream, out-of-frame initiation codon, which may lead to translation termination at a PTC (the normal initiation codon is no longer used). b | Examples of alternative splicing events that can introduce PTCs to mRNAs. The top of each panel shows a schematic representation of a pre-mRNA with exons (boxes) and introns (red lines). Boxes with a red horizontal strikethrough illustrate alternatively spliced exons or introns. The initiation codon (AUG) and termination codon (TC) are indicated and so are premature termination codons (PTC) when in-frame. Productive and NMD-activating pre-mRNA splicing events are indicated by dashed green and red lines, respectively. Green and red arrows point to productively and non-productively (NMD-targeted) spliced mRNA isoforms. Untranslated and translated regions are depicted in light and dark green, respectively. (1) Exon inclusion (shown here as an entire exon) may introduce an in-frame PTC directly (shown here) or indirectly by causing a frameshift. The same can happen following intron retention. The normal termination codon is no longer used. (2) Exon exclusion may introduce a PTC by shifting the reading frame. (3) 
Splicing in the 3' UTR can functionally convert the normal termination codon to a PTC. c Alternative transcription initiation and 3' end processing sites may introduce PTCs. (1) Alternative transcription initiation sites (right-pointing arrows; shown here within the same exon) can direct the usage of an NMD-activating ORF. The downstream initiation codon is no longer used. (2) Alternative 3' end processing (polyadenylation) sites (pA; shown here within the same exon) can change the context of a termination codon thereby potentially activating NMD. 